\title{
Morphology-based black and white filters for topology optimization
}

\author{
Sigmund, Ole
}

Published in:

Structural and Multidisciplinary Optimization

Link to article, DOI:

10.1007/s00158-006-0087-x

Publication date:

2007

Document Version

Early version, also known as pre-print

Link back to DTU Orbit

Citation (APA):

Sigmund, O. (2007). Morphology-based black and white filters for topology optimization. Structural and Multidisciplinary Optimization, 33(4-5), 401-424. https://doi.org/10.1007/s00158-006-0087-x

\section{General rights}

Copyright and moral rights for the publications made accessible in the public portal are retained by the authors and/or other copyright owners and it is a condition of accessing publications that users recognise and abide by the legal requirements associated with these rights.

- Users may download and print one copy of any publication from the public portal for the purpose of private study or research.

- You may not further distribute the material or use it for any profit-making activity or commercial gain

- You may freely distribute the URL identifying the publication in the public portal

If you believe that this document breaches copyright please contact us providing details, and we will remove access to the work immediately and investigate your claim. 


\title{
Morphology-based black and white filters for topology optimization
}

\author{
Ole Sigmund \\ Department of Mechanical Engineering, Solid Mechanics \\ Technical University of Denmark \\ Nils Koppels Alle, B. 404, DK-2800 Lyngby, Denmark \\ Tel.: (+45) 45254256, Fax.: (+45) 45931475 \\ email: sigmund@mek.dtu.dk
}

Lyngby November 9, 2006

\begin{abstract}
In order to ensure manufacturability and mesh-independence in density-based topology optimization schemes it is imperative to use restriction methods. This paper introduces a new class of morphology based restriction schemes which work as density filters, i.e. the physical stiffness of an element is based on a function of the design variables of the neighboring elements. The new filters have the advantage that they eliminate grey scale transitions between solid and void regions. Using different test examples, it is shown that the schemes in general provide black and white designs with minimum length-scale constraints on either or both minimum hole sizes and minimum structural feature sizes. The new schemes are compared with methods and modified methods found in the literature.
\end{abstract}

Keywords: topology optimization, regularization, image processing, morphology operators, manufacturing constraints.

\section{Introduction}

It is well-known that the standard "density approach to topology optimization" (Bendsøe, 1989; Sigmund, 2001a) is prone to problems with checkerboards and mesh-dependency if no regularization scheme is applied. As reviewed by Sigmund and Petersson (1998) a large number of works have suggested different regularization schemes to be used in connection with topology optimization. However, each scheme has its pros and cons and probably the ideal scheme still has to be invented.

In this paper, we present a new family of regularization schemes that are based on image morphology operators. Although the new schemes, as will be demonstrated, solve many of the known problems with existing schemes, they require a continuation approach and hence are not very computationally efficient, thus there is still room left for improvement.

Restriction methods for density based topology optimization problems can roughly be divided into three categories: 1) mesh-independent filtering methods, constituting sensitivity filters (Sigmund, 1994, 1997; Sigmund and Petersson, 1998) and density filters (Bruns and Tortorelli, 2001; Bourdin, 2001); 2) constraint methods such as perimeter control (Ambrosio and Buttazzo, 1993; Haber et al, 1994), global gradient control (Bendsøe, 1995; Borrvall, 2001), local gradient control (Niordson, 1983; Petersson and Sigmund, 1998; Zhou et al, 2001), regularized penalty methods (Borrvall and Petersson, 2001) and integral filtering (Poulsen, 2003); and 3) other methods like wavelet parameterizations (Kim and Yoon, 2000; Poulsen, 2002), phase-field approaches (Bourdin and Chambolle, 2003; Wang and Zhou, 2004) and level-set methods (Sethian and Wiegmann, 2000; Wang et al, 2003; Allaire et al, 
$2004)^{1}$. The filtering methods in group 1 are probably the most popular ones due to their ease of implementation and their efficiency. In general, the constraint methods in group 2 may be difficult to use because they require a problem and geometry dependent choice and tuning of the constraint value. The alternative methods in group 3 are mostly still in their infancy and have yet to be successfully applied to advanced problems with many constraints. There are also methods that are hybrids between categories 1 and 2 (Zhou et al, 2001; Cardoso and Fonseca, 2003). Here, the box-constraints of the optimization algorithm are modified according to a filtering scheme, resulting in local gradient controllike behavior.

The morphology based restriction methods presented in this paper are extensions of the existing density filtering methods. The exact description of the methods will be given later, but a feature of both sensitivity and density based filtering methods is that they result in optimized topologies that have grey (porous material) transition regions between solid and void areas as illustrated in Fig.1a. These transition regions whose width depends on the filter size (i.e. the minimum lengthscale) may be an advantage or a disadvantage depending on the problem considered. In a standard compliance minimization problem, the grey transition regions make it easy to perform an automated post-processing procedure to identify hole shapes and other features by a simple iso-density curve (Fig.1a). Usually, the post-processed structure has a performance that is very close to the optimized structure. However, recently the author's research group has applied the topology optimization to phononic and photonic crystal design (Sigmund and Jensen, 2003; Jensen and Sigmund, 2004) where structures with intricate semi-periodic patterns appear as the result of the topology optimization process. An example of the design of a nano-scale optical splitter from Borel et al (2005) is shown in Fig.1b. Again grey transition regions due to the filtering are observed. However, in this case a simple post-processing based on an iso-density curve and thresh-holding results in a structure with a performance that is degraded and entirely different from the optimized structure. The large difference is attributed to the localized optical wave patterns that can get significantly altered by even small geometrical changes. This as opposed to the minimum compliance case where local stress changes often are ignorable with regards to the global compliance of the structure.

An often used method to obtain crisp boundaries in filter-based topology optimization is to gradually decrease the size and influence of the filter during the optimization process. Whereas this approach in many cases goes well, there are other cases where a mesh-dependent design may re-appear during the continuation approach, and certainly it is impossible to maintain minimum feature sizes (like minimum hole size or minimum bar thicknesses) if the filter effect is diminished.

In conclusion, there is a need for a regularization technique that preserves the simplicity and efficiency of the filtering techniques but at the same time results in discrete and easily interpretable designs that have minimum feature sizes controlled by the filter radius. A number of alternative schemes that attempt to solve these requirements have appeared recently and will be reviewed in the example section. In order to provide a basis for comparison of the new schemes suggested in this paper and the alternatives presented in the literature, a list of requirements for the "ideal restriction method" could be:

1. Mesh-independent and checkerboard-free solutions

2. Black and white $(0 / 1)$ solutions

3. Manufacturability (tooling radius, minimum hole size, etc.)

4. No extra constraints

5. Not too many tuning parameters

\footnotetext{
${ }^{1}$ Level-set methods can be categorized as restriction methods in two dimensions but not in three dimensions where topological changes are allowed.
} 


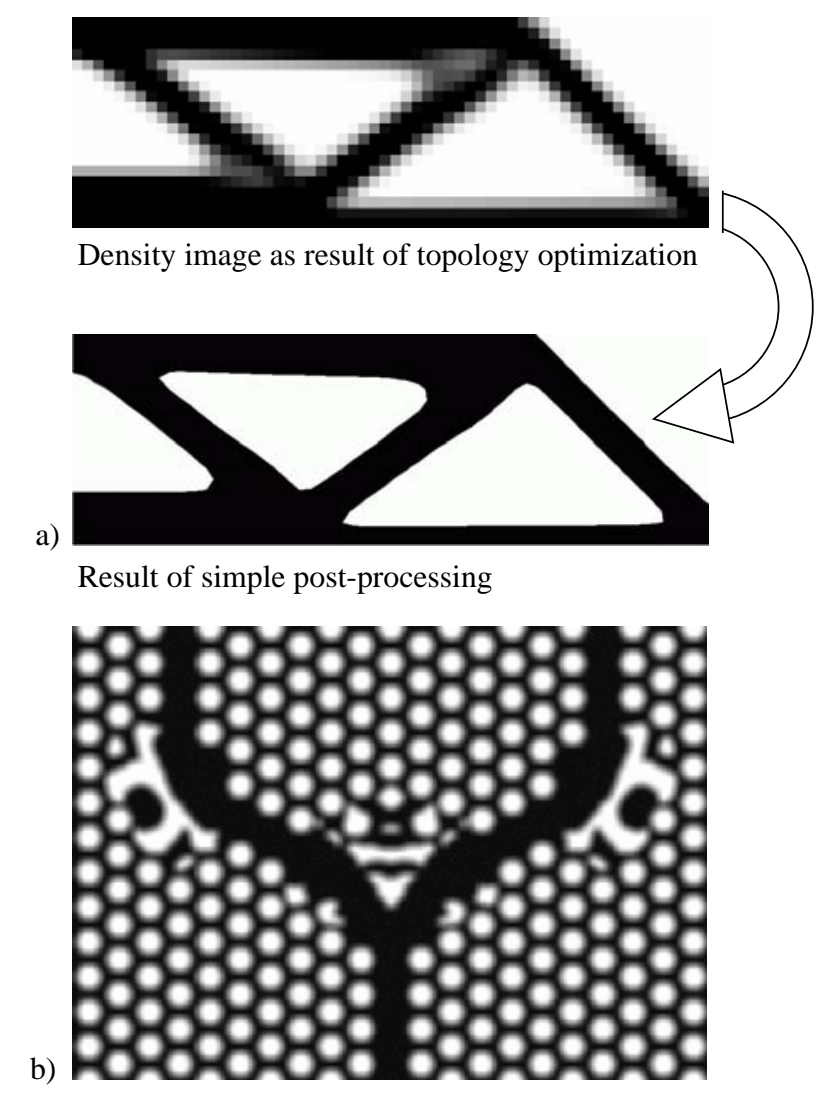

Figure 1: Demonstration of grey (porous) transition regions as results of topology optimization using filtering approaches. a) Grey scale picture of optimized MBB-beam with post-processed version based on the $50 \%$ density contour curve. b) Grey scale picture of a photonic crystal splitter nano-optical device. 
6. Stable and fast convergence

7. General applicability

8. Simple implementation

9. Low CPU-time

Some comments should be attached to the list. Concerning item 1, a good scheme should result in designs with smoothly varying geometries with details defined by the radius of the filter. The need for crisp black and white solution (item 2) was discussed above. Manufacturability (item 3) is closely related to item 1 but also includes the avoidance of non-manufacturable features such as sharp corners. Extra constraints (item 4) require difficult and problem dependent choice of constraint values and should be avoided. Tuning parameters (item 5) that are problem and geometry dependent should be avoided. With "stable convergence" (item 6) it is understood that the method is independent of the problem formulation (and physical setting) and does not require delicate fine-tuning of parameters. With "general applicability" (item 7) it is understood that the scheme should be applicable to problems involving any kind of objective functions, constraints, density interpolation functions and physical models. With "simple implementation" (item 8) it is understood that one can use "black-box" finite element codes and optimizers.

Depending on the viewpoint, the prioritized list may look different. The suggested list probably applies to researchers and academicians who are interested in getting as close to the global optimum as possible whereas software vendors may move "stable and fast convergence" (item 6) and "Low CPU-time" (item 9) up front as items 1 and 2, respectively.

In order to test and compare new and old filter methods this paper suggests three different test problems. When performing a test and review of different filtering methods it is very difficult to perform a fair comparison since every author has his preferred parameter settings, interpolation function, optimizer and test problems. A method may work very well in one problem setting or for one test problem, whereas it may fail in other settings. However, for a method to be generally stable and applicable, it should be possible to use it in a standard setting. Hence, if a method does not work well for the three test cases provided in this paper, it will probably not be working well for other formulations either. The three test cases are therefore not chosen arbitrarily but are carefully selected in order to exploit the weaknesses of the different filtering methods with regards to the 9 requirements to an ideal filter method listed earlier.

The paper is organized as follows. In section 2 we set up the three test problems that will be used to benchmark the performance of new and old filters. In section 3 we review and set up the equations for the original sensitivity and density filters. In section 4 we present a new family of filtering techniques based on image morphology operators and in section 5 we briefly review black and white filtering alternatives from the recent literature. In section 6 we discuss implementation aspects and sensitivity analysis for the new morphology based filter schemes. In section 7 we present, discuss and compare the results obtained for the different approaches. Finally, we summarize our findings and try to draw some general guidelines for the use of filtering methods in density based topology optimization in section 8 .

\section{Problem formulation and definition of test problems}

In general, many new procedures and schemes in topology optimization are tested on simple compliance minimization problems with a material resource constraint. However, it is dangerous to draw general conclusions from such studies since compliance minimization problems have some features that make them especially easy to solve. First of all, the compliance (objective) function is self-adjoint, i.e. 
the direct solution and the adjoint solution are the same (possibly with opposite signs) and thus sensitivities will always have the same sign. Second, the single volume constraint is linear and monotone in the design variables providing for easy solution by optimality criteria approaches or other heuristic procedures. Nevertheless, we do include a compliance based test problem here (the MBB-beam) for comparison with the literature but we also use two other test cases that are harder to solve. In this connection, it should also be noted that some authors have used a two-bar truss example, i.e. a very short cantilever beam, as a test case for filtering schemes. The two-bar example (obviously) has a unique solution (two bars) and thus demonstrating mesh independent solutions for this test case demonstrates nothing.

A problem that is already much more difficult to solve than simple compliance minimization problems is the design of a compliant force inverter (Sigmund, 1997, 2000). Here we use a simple formulation as presented in Sigmund (2000) and Bendsøe and Sigmund (2003) that requires minimization of output displacement subject to a single (linear) constraint on material resource. This problem is non-self adjoint, meaning that the sensitivities of the objective function may take both positive and negative values - a feature that makes the convergence of this problem much more prone to local minima.

The third test case involves wave propagation and has no volume constraint. The latter feature disqualifies many of the existing filter approaches since they rely on an active material resource constraint.

The test cases are all based on the standard "density based approach to topology optimization", i.e. the design variables $\boldsymbol{\rho}$ represent piece-wise constant element densities. We consider linear isotropic materials and the Young's modulus of an element is a function of the element design variable $\rho_{e}$ given by the modified SIMP (Simplified Isotropic Material with Penalization) interpolation scheme

$$
E_{e}=E\left(\rho_{e}\right)=E_{\text {min }}+\rho_{e}^{p}\left(E_{0}-E_{\text {min }}\right), \quad \rho_{e} \in[0,1],
$$

where $p$ is the penalization power, $E_{\min }$ is the stiffness of soft (void) material (non-zero in order to avoid singularity of the stiffness matrix) and $E_{0}$ is the Young's modulus of solid material. Use of the modified SIMP scheme (1) as opposed to the standard SIMP scheme $E\left(\rho_{e}\right)=\rho_{e}^{p} E_{0}$ with $\left.\left.\rho_{e} \in\right] 0,1\right]$ has a number of advantages, including that the minimum stiffness value is independent of the penalization factor, that the modified formulation also covers two phase design problems and that the modified form is easier to generalize for use in the various filtering schemes discussed in the present paper. The test structures are all discretized by 4-node bi-linear finite elements. Otherwise, the implementation is done in MATLAB as described in Sigmund (2001a). As the optimizer we use the MATLAB implementation of the Method of Moving Asymptotes (Svanberg, 1987) made freely available for research purposes by Krister Svanberg.

In the following we define the three problem specific formulations.

\subsection{The MBB-beam}

The optimization problem for the MBB-beam example may be written as

$$
\left.\begin{array}{rl}
\min _{\boldsymbol{\rho}}: & f(\boldsymbol{\rho})=\mathbf{U}^{T} \mathbf{K} \mathbf{U}=\sum_{e} \mathbf{u}_{e}^{T} \mathbf{k}_{e} \mathbf{u}_{e} \\
\text { s.t. : } & \mathbf{K U}=\mathbf{F} \\
: & g=V(\boldsymbol{\rho}) / V^{*}-1=\sum_{e} v_{e} \rho_{e} / V^{*}-1 \leq 0 \\
: & \mathbf{0} \leq \boldsymbol{\rho} \leq \mathbf{1}
\end{array}\right\},
$$

where $\mathbf{K}, \mathbf{U}$ and $\mathbf{F}$ are the global stiffness matrix, displacement vector and force vector, respectively, lower case symbols indicate element wise quantities, $\mathbf{k}_{e}=\mathbf{k}\left(\rho_{e}\right)=E\left(\rho_{e}\right) \mathbf{k}_{e}^{0}$, is the element stiffness matrix, $\mathbf{k}_{e}^{0}$ is the element stiffness matrix for unit Young's modulus, the sum symbol in the first line indicates finite element summation, $V^{*}$ is the material resource constraint and $v_{e}$ is the volume of 
element $e$. The sensitivity expressions are simply found as

$$
\begin{aligned}
& \frac{\partial f}{\partial \rho_{e}}=-\mathbf{u}_{e}^{T} \frac{\partial \mathbf{k}_{e}}{\partial \rho_{e}} \mathbf{u}_{e} \\
& \frac{\partial \mathbf{k}_{e}}{\partial \rho_{e}}=p\left(E_{0}-E_{\min }\right) \rho_{e}^{p-1} \mathbf{k}_{e}^{0} \\
& \frac{\partial g}{\partial \rho_{e}}=v_{e} / V^{*}
\end{aligned}
$$

The design domain and its dimensions are shown in Fig. 2a. Due to symmetry we model only half the design domain which is discretized with 120 by 40 bi-linear quadrilaterals. The Young's modulus of solid material is $E_{0}=1$, the minimum stiffness is $E_{\text {min }}=10^{-9}$, the Poisson's ratio is $\nu=0.3$, the filter radius (see later) is $R=3.5$ and the penalization factor is $p=3$.

\subsection{The compliant force inverter}

In compliant mechanism design, a typical design goal is to transfer work from an input actuator to an output spring (cf. Sigmund, 2000). For the present case, we consider the force inverter that previously has been used as a benchmark (Sigmund, 1997). The optimization problem for the force inverter example may be written as

$$
\left.\begin{array}{rl}
\min _{\boldsymbol{\rho}}: & f(\boldsymbol{\rho})=\mathbf{L}^{T} \mathbf{U} \\
\text { s.t. : } & \mathbf{K U}=\mathbf{F} \\
: & g=V(\boldsymbol{\rho}) / V^{*}-1=\sum_{e} v_{e} \rho_{e} / V^{*}-1 \leq 0 \\
: & \mathbf{0} \leq \boldsymbol{\rho} \leq \mathbf{1}
\end{array}\right\},
$$

where $\mathbf{L}$ is a unit length vector with zeros at all degrees of freedom except at the output point where it is one. The sensitivity is simply found as

$$
\frac{\partial f}{\partial \rho_{e}}=\boldsymbol{\lambda}_{e}^{T} \frac{\partial \mathbf{k}_{e}}{\partial \rho_{e}} \mathbf{u}_{e},
$$

where $\boldsymbol{\lambda}$ is the global adjoint vector found by the solution of the adjoint problem $\mathbf{K} \boldsymbol{\lambda}=-\mathbf{L}$ and $\boldsymbol{\lambda}_{e}$ is the part of the adjoint vector associated with element $e$. The design domain and its dimensions are shown in Fig. 2b. For faster computations we consider only half the structure due to symmetry. The design domain is discretized with 120 by 60 bi-linear quadrilaterals, the filter radius is $R=2.5$, the input force is $F_{\text {in }}=1$ and the input and output spring stiffnesses are $k_{\text {in }}=1$ and $k_{\text {out }}=0.001$, respectively. Otherwise, the parameters are the same as for the MBB example. Remark that the input and output spring stiffnesses are chosen such that the resulting mechanism without filtering exhibits so-called one-node-connected hinges. These are numerical artifacts related to checkerboard patterns which for a compliant mechanism have the (artificial) advantage that they are good in transmitting forces but deliver no resistance to bending. For higher stiffness of the output spring, the hinge-like connection becomes more solid (distributed compliant) on the cost of smaller output displacement (Sigmund, 1997). In order to compare the different filtering methods abilities to prevent hinges, we here select a small value of the output spring stiffness.

\subsection{The wave transmitter}

Recently, the topology optimization method has been applied to the design of wave-propagation problems based on band gap structures both for phononic and photonic applications (Sigmund and Jensen, 2003; Jensen and Sigmund, 2004). A special feature of these problems is that they require no volume constraint. An optimization problem for a wave transmitter example may be written as

$$
\left.\begin{array}{cl}
\min _{\boldsymbol{\rho}}: & f(\boldsymbol{\rho})=-\mathbf{U}^{T} \mathbf{L} \overline{\mathbf{U}} \\
\text { s.t. : } & \left(\mathbf{K}+i \Omega \mathbf{C}-\Omega^{2} \mathbf{M}\right) \mathbf{U}=\mathbf{F} \\
: & \mathbf{0} \leq \boldsymbol{\rho} \leq \mathbf{1}
\end{array}\right\},
$$




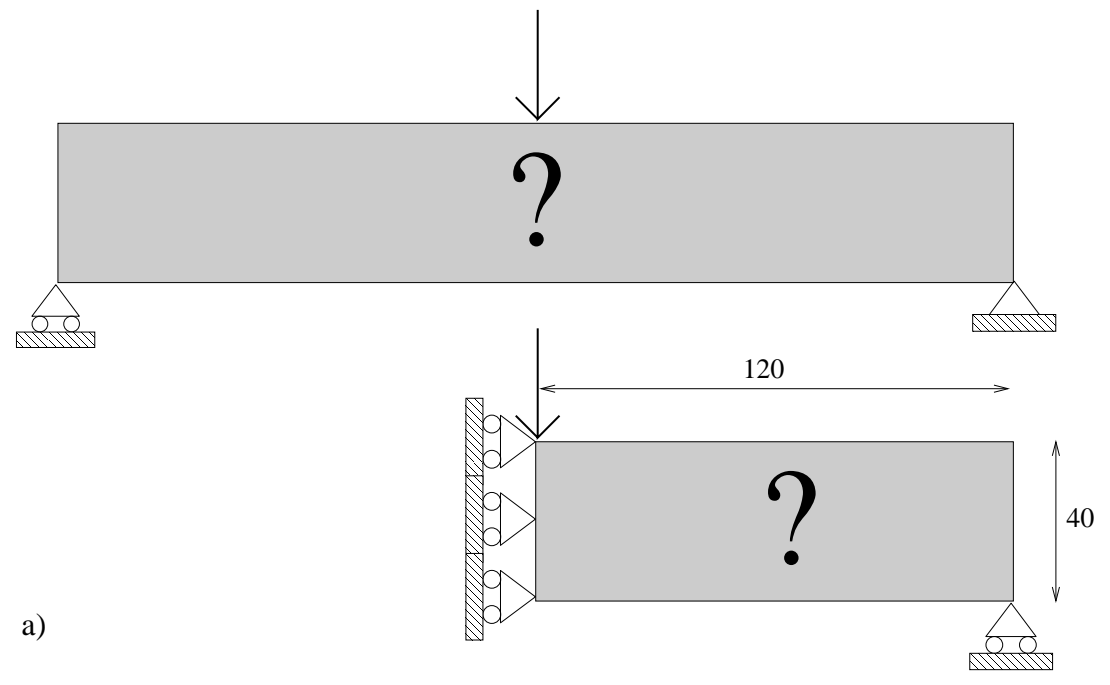

b)

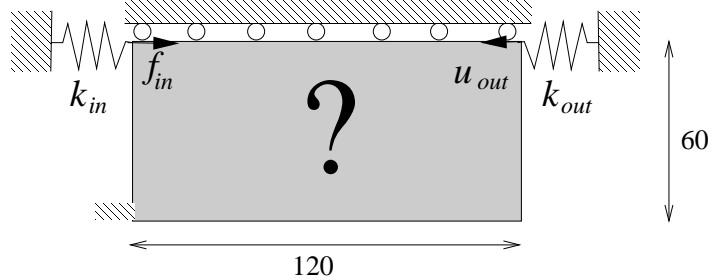

c)

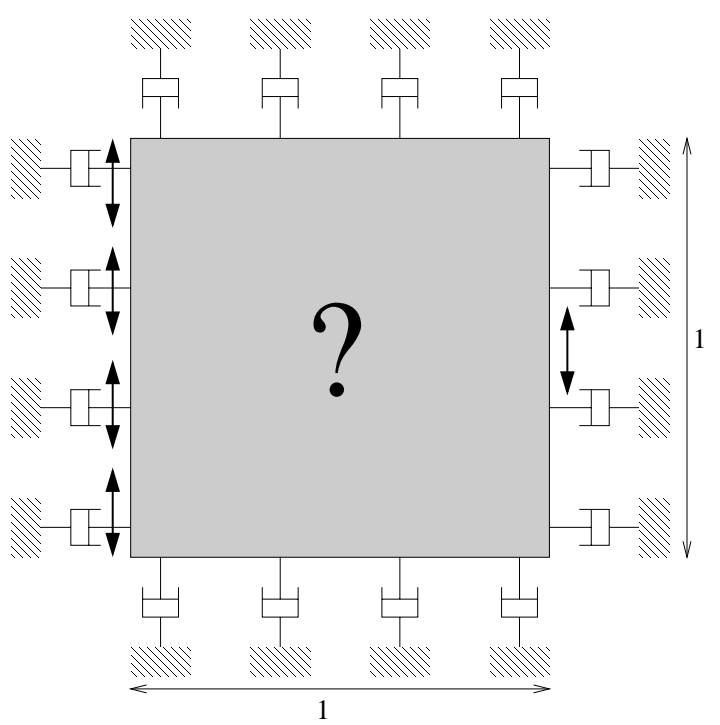

Figure 2: Design domains and boundary conditions for the three test problems. a) The MBB-beam, b) the compliant force inverter and c) a wave focussing device 
where $\mathbf{L}$ this time is a zero matrix with ones at the diagonal entries corresponding to the degrees of freedom where the wave amplitude is to be maximized, $i$ is the complex operator and overbars mean complex conjugate. $\mathbf{M}$ and $\mathbf{C}$ are the global mass and damping matrices, respectively. The global mass matrix is defined as $\mathbf{M}=\sum_{e}\left(\varrho_{\text {min }}+\rho_{e}\left(\varrho_{0}-\varrho_{\text {min }}\right)\right) \mathbf{m}_{e}^{0}$, where $\varrho_{\text {min }}$ and $\varrho_{0}$ are the specific mass densities of background material and foreground material, respectively, and $\mathbf{m}_{e}^{0}$ is the element mass matrix for unit specific mass density. In order to assure black and white solutions we apply the so-called "pamping" (penalization damping) method introduced in Jensen and Sigmund (2005). The global damping matrix is defined as $\mathbf{C}=\sum_{e} q \rho_{e}\left(1-\rho_{e}\right) \mathbf{c}_{e}^{0}$, where $q$ is the pamping factor and $\mathbf{c}_{e}^{0}$ is the element damping matrix (here mass proportional, i.e. $\mathbf{c}_{e}^{0}=\mathbf{m}_{e}^{0}$ ). The pamping method ensures black and white solutions in wave transmission problems by introducing an (artificial) damping in intermediate density elements.

The sensitivity of the objective function is found as

$$
\begin{aligned}
& \frac{\partial f}{\partial \rho_{e}}=2 \Re\left(\boldsymbol{\lambda}_{e}^{T}\left[\frac{\partial \mathbf{k}_{e}}{\partial \rho_{e}}+i \Omega \frac{\partial \mathbf{c}_{e}}{\partial \rho_{e}}-\Omega^{2} \frac{\partial \mathbf{m}_{e}}{\partial \rho_{e}}\right] \mathbf{u}_{e}\right), \\
& \frac{\partial \mathbf{c}_{e}}{\partial \rho_{e}}=q\left(1-2 \rho_{e}\right) \mathbf{m}_{e}^{0}, \\
& \frac{\partial \mathbf{m}_{e}}{\partial \rho_{e}}=\left(\varrho_{0}-\varrho_{\min }\right) \mathbf{m}_{e}^{0}
\end{aligned}
$$

where $\boldsymbol{\lambda}$ is the solution to the adjoint problem $\left(\mathbf{K}+i \Omega \mathbf{C}-\Omega^{2} \mathbf{M}\right)^{T} \boldsymbol{\lambda}=-\mathbf{L} \overline{\mathbf{U}}$ and $\Re(\cdot)$ denotes the real part of $(\cdot)$. In practice, the objective function for wave propagation problems may change by orders of magnitude during optimization which may cause normalization problems for the optimizer. Therefore, we optimize $\log (f)$ instead and the sensitivities are modified accordingly (i.e. $\partial f / \partial \rho_{e}$ becomes $\left.\left(\partial f / \partial \rho_{e}\right) / f\right)$.

The design domain and its dimensions are shown in Fig. 2c. The design domain is discretized with 80 by 80 bi-linear quadrilaterals, the filter radius is $R=2.5 / 80$, the pamping coefficient is $q=5$ and the angular frequency is $\Omega=10$. The material properties used mimic the stiffness and density ratios of steel and polyester, i.e. $E_{0}=1, E_{\min }=0.025, \varrho_{0}=1$ and $\varrho_{\min }=0.15$. The structure is supported by dampers perpendicular to the surfaces at all four edges with damping factors 0.1 and a distributed vertical input load (i.e. an incoming shear wave) on the left edge. The goal of the optimization problem is to maximize the vertical amplitude at the center node of the right edge, i.e. to obtain the most efficient shear wave focussing device.

\section{Basic filtering methods}

As discussed in the introduction, filtering methods intended for regularization of topology optimization problems can be divided into density and sensitivity based methods. In the former case, each element density is redefined as a weighted average of the densities in a mesh-independent neighborhood of the element, before calling the finite element solver, and afterwards the sensitivities are modified in a consistent way. In the latter case, the finite element problem is solved in the standard way but after calculating the sensitivities consistently, they are heuristically modified as weighted averages of the sensitivities in mesh-independent neighborhoods.

The neighborhood of element $e$, here named $N_{e}$, is generally specified by the elements that have centers within a given filter radius $R$ of the center of element $e$, i.e.

$$
N_{e}=\left\{i \mid\left\|\mathbf{x}_{i}-\mathbf{x}_{e}\right\| \leq R\right\},
$$

where $\mathbf{x}_{i}$ is the spatial (center) location of element $i$.

In order to compare the different methods we perform different simplifications and generalizations. First, all methods are described in a discrete setting and density and sensitivity information is assumed constant within each element and is evaluated in element centers. It is clear that especially for small 
filter radius to mesh size ratios this simplification may cause unwanted effects that may distort the conclusions for the numerical results. Second, some of the filters from the literature are based on density interpolation schemes being different from piece-wise constant. Again, in order to make a fair and general comparison, these schemes are reformulated in the standard piece-wise constant density form. It may be that this reformulation causes not so favorable behavior of the method, however, in order to be generally applicable, the filtering method should also work in this setting.

A pitfall when working with filtering methods is the treatment of mesh boundaries. In principle one should expand the filter and include elements outside the mesh at external edges as void elements and likewise one should expand the filter outside the mesh at symmetry edges and mirror the values from inside the mesh. Furthermore, supported or non-zero-traction boundaries should be specified to be solid. In this way, one would obtain consistent filtering and avoid problems at edges and corners. However, no papers appear to have implemented such a scheme. Instead, it seems to be most common simply to perform the filtering operations only based on the elements inside the mesh. This is also the scheme applied here. As will be discussed later, the effect of the simplification seems to vary from filter scheme to filter scheme.

In the following two subsections, we discuss the two different filtering approaches in more detail.

\subsection{Density filtering}

Density filters work by modifying the element density and thereby stiffness to be a function of the densities in a specified neighborhood of an element. The modified element density can be written as

$$
\tilde{\rho}_{e}=\tilde{\rho}_{e}\left(\rho_{i \in N_{e}}\right)
$$

i.e., the modified element density $\tilde{\rho}_{e}$ is a function of the neighboring design variables $\rho_{i \in N_{e}}$. Hence, the modified stiffness in element $e$ can be written as

$$
E_{e}=E_{e}(\boldsymbol{\rho})=E_{e}\left(\tilde{\rho}_{e}\right)=E_{\min }+\tilde{\rho}_{e}^{p}\left(E_{0}-E_{\min }\right),
$$

where $\tilde{\rho}_{e}$ is the filtered density. Several ways for implementing the density filtering have been proposed in the literature and the main ones will be reviewed below. Alternative density filter schemes will be reviewed in section 5 .

An important characteristic of filter operators is volume-preservation, i.e. the volume of material should be the same before and after the filtering process. In practice, exact volume preservation is seldomly fulfilled due to boundary influence. Some of the suggested variations of the density filter that have appeared in the literature do not preserve volume. This is no problem as long as the volume fraction constraint is modified accordingly and there are no fixed solid or void regions in the design domain. For the present case, we are operating with a modified density function, hence the volume constraint must also be imposed on the modified (physical) density field. This means that the volume fraction constraint for a density filter approach must be defined as

$$
g=V(\tilde{\boldsymbol{\rho}}) / V^{*}-1=\sum_{e} v_{e} \tilde{\rho}_{e} / V^{*}-1 \leq 0
$$

and not as $g=\sum_{e} v_{e} \rho_{e} / V^{*}-1$ as erroneously defined in some papers. The problem with non-volume preserving filters and fixed solid or void regions will be discussed in the example section. 


\section{Basic density filtering}

Density filtering was introduced by Bruns and Tortorelli (2001) and mathematically proven as a viable approach by Bourdin (2001). The filtered density measure is

$$
\tilde{\rho}_{e}=\frac{\sum_{i \in N_{e}} w\left(\mathbf{x}_{i}\right) v_{i} \rho_{i}}{\sum_{i \in N_{e}} w\left(\mathbf{x}_{i}\right) v_{i}},
$$

where the weighting function $w\left(\mathbf{x}_{i}\right)$ is given by the linearly decaying (cone-shape) function

$$
w\left(\mathbf{x}_{i}\right)=R-\left\|\mathbf{x}_{i}-\mathbf{x}_{e}\right\|
$$

as suggested in Bruns and Tortorelli (2001); Bourdin (2001). As defined earlier, $v_{i}$ denotes the volume of element $i$.

In order to obtain a smoother weighting function, the Gaussian (bell shape) distribution function

$$
w\left(\mathbf{x}_{i}\right)=e^{-\frac{1}{2}\left(\frac{\left\|\mathbf{x}_{i}-\mathbf{x}_{e}\right\|}{\sigma_{d}}\right)^{2}}
$$

was suggested in Bruns and Tortorelli (2003); Wang and Wang (2005) for use with the density filtering (12). In Bruns and Tortorelli (2003) the variance is defined by $\sigma_{d}=R / 3$ and in Wang and Wang (2005) by $\sigma_{d}=R / 2$. In both cases, the filter is truncated at radius $R$. For the latter case, it means that the bell-shape curve is heavily truncated. In tests, the author did not experience any advantages of the smooth Gaussian function compared to the original linear function, however, it is possible that there is an advantage when the filtering scheme is used in connection with the element removal and re-introduction scheme suggested by Bruns and Tortorelli (2003).

For completeness one may also consider a constant weighting function

$$
w\left(\mathbf{x}_{i}\right)=1 .
$$

together with the density filtering (12).

The effect of the weighting is highest for the constant weighting (15), less for the linear decaying weighting (13) and least for the Gaussian weighting (14). From a computing time point of view it is thus more efficient to use the constant weighting function (15) since a smaller filter radius will result in the same filtering effect. In the comparison studies of the present paper, we will only present result for the linearly decaying weighting case (13).

When using density filters it is important to do the physically correct interpretation of the design. In the literature, this has sometimes caused problems although it should be rather simple. The original design variables $\boldsymbol{\rho}$ have no physical meaning! They are only used as intermediate mathematical variables. Therefore, one should always show plots of the filtered design variables $\tilde{\boldsymbol{\rho}}$ (the densities used in the SIMP-interpolation (10)), since they represent the physical density of the elements. One could also plot $\tilde{\boldsymbol{\rho}}^{p}$ which would represent the physical stiffness of the elements. For historical reasons we prefer the former case (i.e. we will plot the filtered densities $\tilde{\boldsymbol{\rho}}$ ) but we also include plots of the (non-physical) design variable fields $\rho$ for illustrative purposes.

\subsection{Sensitivity filtering}

Sensitivity filtering was introduced by Sigmund (1994, 1997); Sigmund and Petersson (1998) and has become very popular both in academia and in commercial programs. The main idea is to base design updates on filtered sensitivities instead of the real sensitivities. Obviously, this is a simple but 
potentially risky approach, especially for line-search based optimization schemes since the sensitivity data may not represent a descent direction and hence the optimization may stop prematurely. However, numerous applications and multiple physics settings have proven the method very robust and reliable using most of the popular optimization tools.

\section{Original mesh-independent sensitivity filter}

The original form of the sensitivity filter presented by Sigmund (1997) was

$$
\frac{\widetilde{\partial f}}{\partial \rho_{e}}=\frac{\sum_{i \in N_{e}} w\left(\mathbf{x}_{i}\right) \rho_{i} \frac{\partial f}{\partial \rho_{i}}}{\rho_{e} \sum_{i \in N_{e}} w\left(\mathbf{x}_{i}\right)},
$$

where the weighting function $w\left(\mathbf{x}_{i}\right)$ is the linearly decaying function (13). When the lower bound on the design variable is 0 as it is here, $\rho_{e}$ in the denominator of (16) should be substituted with $\max \left(\rho_{e}, \varepsilon\right)$ where $\varepsilon$ is a small number (e.g. $10^{-3}$ ).

A modification that accounts for non-regular meshes with varying element volumes $v_{e}$ is

$$
\frac{\widetilde{\partial f}}{\partial \rho_{e}}=\frac{\sum_{i \in N_{e}} w\left(\mathbf{x}_{i}\right) \rho_{i} \frac{\partial f}{\partial \rho_{i}} / v_{i}}{\rho_{e} / v_{e} \sum_{i \in N_{e}} w\left(\mathbf{x}_{i}\right)} .
$$

Intuitively, it would have made more sense to multiply by the element volumes (big elements, big weight and small elements, small weight), however, the motivation for dividing by the volumes is partly based on numerical experiments and partly based on energy considerations. By dividing by the element volume, the term inside the summation in the numerator can be interpreted as an averaged strain energy density (energy per unit volume).

Through time, several researchers have tried to explain the exact workwise of the sensitivity filter, however, so far without success. Since the sensitivities are modified heuristically, it is probably impossible to figure out what objective function is actually being minimized but generally it may be stated that the filtered sensitivities correspond to the sensitivities of a smoothed version of the original objective function. Despite that the sensitivities are modified in a heuristic way, the author has throughout his career solved numerous different problems involving up to 20 filtered constraints without convergence problems. A key point behind the success of the method seems to be the scaling with the density in the nominator and the denominator in (16). A number of modifications of the filter will be discussed in section 5 .

\section{Filtering based on image morphology operators}

A common problem for the standard density and sensitivity filters discussed in the previous section is that a grey transition region between solid and void areas always will appear due to the averaging nature of the density filter operator. In the following, we will present a new scheme that prevents the grey transition regions but (mostly) preserves the good features of the density filtering method.

In image analysis, morphology operators are used to quantify holes or objects, restore noisy pictures and perform automatic inspection of image data (Pratt, 1991). The basic morphology operators are "erode" and "dilate" and several extensions can be performed by sequentially applying the erode and dilate operators. 
The work-wise of some basic binary morphology operators are illustrated in the following. An original binary image is shown in Fig. 3a. In image processing one defines a so-called "structuring element" which basically corresponds to the neighborhood operator defined in (8). Performing the morphology operation called erode, verbally corresponds to translating the center of the neighborhood over each element in the design domain. If any of the pixels covered by the neighborhood is void, then the center pixel is made void. Oppositely, the dilate operation sets the center pixel to solid if any pixel covered by the neighborhood is solid. The "open" and "close" operations are defined as an erode followed by a dilate operation and a dilate followed by an erode operation, respectively. The results of the four different operations are seen in Fig. 3b-e. The erode and dilate operators are not volume preserving. In particular, the erode operator removes any feature in the original image that is smaller than the neighborhood (Fig. 3b). Oppositely, the dilate operator fills any hole that is smaller than the neighborhood (Fig. 3c). The open and close operators are volume preserving, have almost the same behaviors as the erode and dilate operators, respectively, but retain the feature sizes above the filter limit from the original image. Immediately, one recognizes that the morphology operators are well suited to provide for feature size control in topology optimization, however, in order to make them practically applicable, the min/max-type operators need to be redefined as continuous and differentiable functions. This is discussed in the following.

\section{Dilate}

In its discrete form the dilation operator is a max operator, i.e. the physical density of element $e$ takes the maximum of the densities in the neighborhood $N_{e}$. The max-formulation is not suitable for gradient-based optimization hence it must be converted to a continuous form. A continuous form that uses the Kreisselmeier and Steinhauser (1983) formulation can be formulated as

$$
\tilde{\rho}_{e}=\log \left(\frac{\sum_{i \in N_{e}} e^{\beta \rho_{i}}}{\sum_{i \in N_{e}} 1}\right) / \beta .
$$

For $\beta$ approaching zero, the filter corresponds to the original density filter (12) with constant weighting (15) and for $\beta$ approaching infinity it corresponds to the max-operator. In order to obtain a numerically stable scheme $\beta$ must be gradually increased using a continuation scheme. Based on numerical experience, the continuation method is started with $\beta=0.2$ and then gradually increased to 200 . Note also that there is no volume dependence in the continuous morphology operator (18) due to its original max-operator nature.

It should also be noted here that the proposed continuation method maintains the filtering effect and is only used to enforce black and white solutions. This is in contrast to other continuation methods where the filtering effect gradually is diminished urging the design into a local (black and white) minimum. As will be seen in the example section, the filter works as a "fixed radius deposition tool", i.e. no details of the structure are smaller than a circular (deposition) tool with radius $R$.

As will be seen later, the resulting physical density distributions $\overline{\boldsymbol{\rho}}$ become quite nice and clear but the extremely thin (has a low volume fraction) design variable structure $\boldsymbol{\rho}$ makes the physical density distribution very sensitive to even small variations in the design variables and may explain occasional instabilities during convergence.

\section{Erode}

The morphological opposite of dilation is erosion, which in its discrete form corresponds to a minoperator, i.e. the physical density of element $e$ is the minimum of the densities in the neighborhood. 
a)

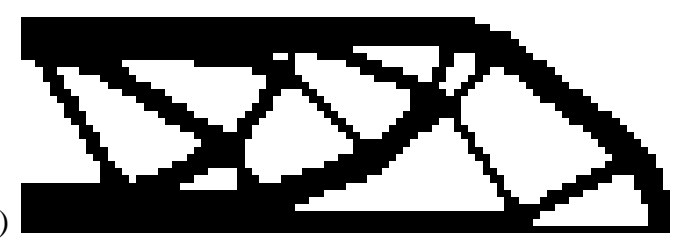

Original image

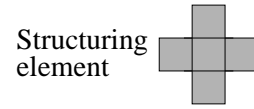

b)

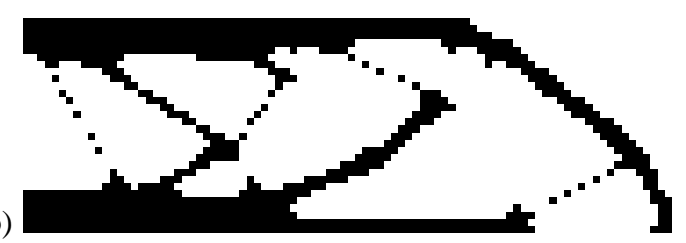

Erode

c)

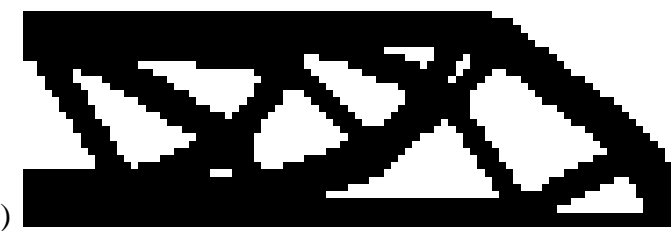

Dilate

d)

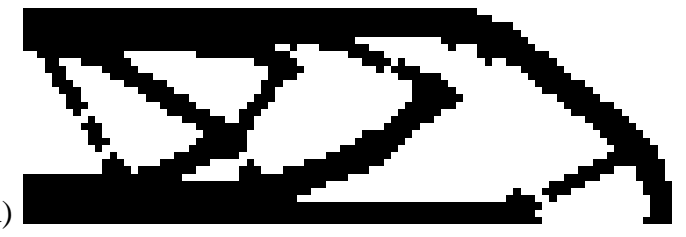

e)

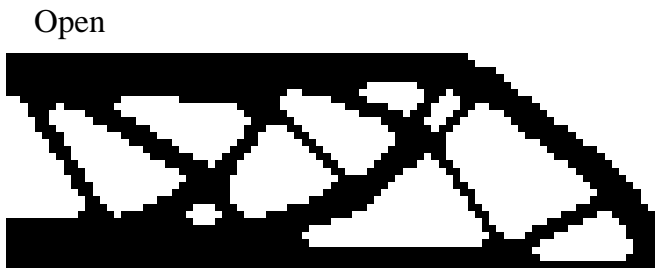

Close

Figure 3: Demonstration of basic image morphology operators. The size of the structuring element is enlarged for illustrative purposes. a) Original image and 5 element structuring element (neighborhood). b) Result of erosion, c) result of dilation, d) result of open (erosion followed by dilation) and e) result of close (dilation followed by erosion). 
As opposed to the dilation operator, the design variable field is very thick (has a high volume fraction) and thus less sensitive to variations in the design variables. The Kreisselmeier-Steinhauser formulation again gives a continuous form of the min-operator

$$
\bar{\rho}_{e}=1-\log \left(\frac{\sum_{i \in N_{e}} e^{\beta\left(1-\rho_{i}\right)}}{\sum_{i \in N_{e}} 1}\right) / \beta .
$$

As before, the filter is implemented by a continuation approach. Also, the filter corresponds to the original density filter (12) with constant weights (15) for $\beta$ approaching zero and to the min operator for $\beta$ approaching infinity. Again, the continuation method starts with $\beta=0.2$ and gradually increases to 200. Also, as will be seen in the examples, this filter works as a "machining constraint", i.e. the resulting topology has no hole features that are smaller than a circle with radius $R$.

Neither the dilation filter nor the erosion filter are volume preserving. A small extension, however, resolves this issue as will be shown in the following.

\section{Close}

Dilation may be followed by erosion to form a morphological close operation. In image processing, this corresponds to filling in all details with dimensions smaller than the filter size. If we denote the dilation operation by tilde and the erosion operation with an overline, the equation for the filtered density becomes

$$
\overline{\tilde{\rho}}_{e}=\overline{\tilde{\rho}}_{e}\left(\tilde{\rho}_{j \in N_{e}}\left(\rho_{i \in N_{j}}\right)\right) .
$$

Results obtained using the close filter are quite similar to those obtained using the dilation filter although the former seems to be a bit more stable due to the denser design variable field. The close operator is volume preserving.

\section{Open}

Likewise, erosion may be followed by dilation to form the open operator. In image processing, this corresponds to removing all details with dimensions smaller than the filter size. If we denote the dilation operation by tilde and the erosion operation with a bar, then the filtered density becomes

$$
\tilde{\bar{\rho}}_{e}=\tilde{\bar{\rho}}_{e}\left(\bar{\rho}_{j \in N_{e}}\left(\rho_{i \in N_{j}}\right)\right) .
$$

Results obtained using the open filter are quite similar to those obtained using the erosion filter although the latter seems to be a bit more stable due to the denser design variable field. The open operator is also volume preserving.

\section{Further morphological operators}

In order to obtain minimum hole size and minimum structural detail size simultaneously, one may combine the open and close operators to form the close-open operator

$$
\tilde{\overline{\tilde{\rho}}}_{e}=\tilde{\overline{\tilde{\rho}}}_{e}\left(\overline{\tilde{\tilde{\rho}}}_{i \in N_{e}}\left(\overline{\tilde{\rho}}_{j \in N_{i}}\left(\tilde{\rho}_{k \in N_{j}}\left(\rho_{l \in N_{k}}\right)\right)\right)\right) \text {, }
$$

or the open-close operator

$$
\overline{\tilde{\tilde{\rho}}}_{e}=\overline{\tilde{\tilde{\rho}}}_{e}\left(\tilde{\bar{\rho}}_{i \in N_{e}}\left(\tilde{\bar{\rho}}_{j \in N_{i}}\left(\bar{\rho}_{k \in N_{j}}\left(\rho_{l \in N_{k}}\right)\right)\right)\right),
$$


i.e., in both cases one has to perform four consecutive filter operations which in itself is not bad, however, the computational burden of the associated sensitivity analysis becomes immense. In practice, the sensitivity of one element becomes a function of its neighbors, which are function of their neighbors, and so on four times. This overwhelming task is much heavier than the solution of the finite element problem (see later discussion). A shortcut may be initially to combine the open and close operators by taking the average of them, i.e.

$$
\overline{\tilde{\tilde{\rho}}}_{e} \approx\left[\overline{\tilde{\rho}}_{e}+\tilde{\bar{\rho}}_{e}\right] / 2=\left[\overline{\tilde{\rho}}_{e}\left(\tilde{\rho}_{j \in N_{e}}\left(\rho_{i \in N_{j}}\right)\right)+\tilde{\bar{\rho}}_{e}\left(\bar{\rho}_{j \in N_{e}}\left(\rho_{i \in N_{j}}\right)\right)\right] / 2 .
$$

After convergence, one may use the real open-close or close-open operators to provide the final solution. The open-close, the close-open and the combination operator are all volume preserving.

\section{Alternative black and white filtering schemes}

Since the review article by Sigmund and Petersson (1998) a number of alternative filtering schemes that claim black and white solutions have been published. In the following, we briefly review these methods. The methods are again categorized in density and sensitivity based methods.

\subsection{Density filters}

\section{Modified density filtering}

In order to obtain more discrete solutions than provided by the original density filter (12), Guo and $\mathrm{Gu}$ (2004) have suggested a modification to (10) where they multiply the filtered density with the original variable, i.e.

$$
E_{e}=E_{e}(\boldsymbol{\rho})=E_{e}\left(\rho, \tilde{\rho}_{e}\right)=E_{\min }+\rho_{e} \tilde{\rho}_{e}^{p}\left(E_{0}-E_{\min }\right),
$$

where $\tilde{\rho}_{e}$ is given by (12) and the linear weighting function (13). The authors claim that their modification results in improved black and white (0/1) solutions, however, they only show pictures based on the design variables $\boldsymbol{\rho}$ which have no physical meaning. For density filtering methods, the vector $\boldsymbol{\rho}$ should only be seen as a mathematical design variable; the physical density distribution to be plotted is given by the filtered vector $\tilde{\boldsymbol{\rho}}$. When the filtered densities are plotted for the examples from the paper by Guo and Gu (2004) many grey elements are observed, hence the basic idea of the method fails. There are two other problems with Guo and Gu's implementation. First, they use the integral over the design variable as volume constraint which again does not make physical sense since their filter is non-volume preserving. However, the correct choice for the material volume constraint is not obvious for this case - but should probably be based on $\sum_{e} v_{e} \rho_{e} \tilde{\rho}_{e}$. Second, the filter does not converge to the original SIMP scheme (10) for the radius $R$ going to zero - it actually converges to $\rho_{e}^{p+1}$. Due to these shortcomings, no results will be shown for this method.

\section{Bi-lateral density filtering}

Wang and Wang (2005) suggest a modification to (12), a so-called bi-lateral filter, which apart from the distance weighting also includes a density weighting

$$
\tilde{\rho}_{e}=\frac{\sum_{i \in N_{e}} w\left(\mathbf{x}_{i}\right) \tilde{w}\left(\rho_{i}\right) v_{i} \rho_{i}}{\sum_{i \in N_{e}} w\left(\mathbf{x}_{i}\right) \tilde{w}\left(\rho_{i}\right) v_{i}},
$$


where

$$
\tilde{w}\left(\rho_{i}\right)=e^{-\frac{1}{2}\left(\frac{\rho_{i}-\rho_{e}}{\sigma_{r}}\right)^{2}} .
$$

Here, the variance factor for the density dependent weighting function $\tilde{w}$ is $\left.\left.\sigma_{r} \in\right] 0,1\right]$. For the case of $\sigma_{r}=\infty$, this filter corresponds exactly to the original density filter (12) with Gaussian weighting (14) and for $\sigma_{r}$ approaching zero, the effect of the filter is eliminated. Despite lots of effort it was not possible to reproduce the results of the paper by Wang and Wang (2005). The authors write that they use a continuation approach to obtain their results, however, no details are given concerning its implementation. In the present investigation the bi-lateral filter is implemented with a fixed value of $\sigma_{r}=1$. A larger value causes further blurring of boundaries corresponding to the original Gaussian density filter (14) and a smaller value causes appearance of checkerboard patterns and meshdependency.

\section{Density filtering with a Heaviside step function}

In order to obtain 0/1 solutions, Guest et al (2004) have modified the original filter (12) with a Heaviside function such that if $\tilde{\rho}_{e}>0$ then the physical element density will become one and only if the filtered density $\tilde{\rho}_{e}=0$ will the physical density be zero. The Heaviside function is approximated as a smooth function governed by the parameter $\beta$, thus the physical density $\bar{\rho}_{e}$ becomes

$$
\bar{\rho}_{e}=1-e^{-\beta \tilde{\rho}_{e}}+\tilde{\rho}_{e} e^{-\beta} .
$$

For $\beta$ equal to zero, this filter corresponds exactly to the original density filter (12) with linearly decaying weighting (13). For $\beta$ approaching infinity, the modification efficiently behaves as a maxoperator, i.e. the density value of the center pixel is set equal to one if any of the pixels within the neighborhood is larger than zero. Note that Guest et al (2004) include an extra term in (28) in order to ensure that the lower bound on the design variable is fulfilled. With the present modified SIMP interpolation scheme (10), this extra term can be left out. It should also be noted that Guest et al (2004) propose to use a more elaborate scheme for the density interpolation. Instead of calculating the filtered density based on the element values within the neighborhood, the paper suggest to use mapped nodal design variables, which is reported to result in better numerical behavior. In order to make a fair comparison of methods we here chose to use the standard element density approach. However, one may keep the idea of Guest et al (2004) in mind for improving convergence also for the new morphology based schemes presented in this paper. The backside of the idea is the more complex implementation that requires numerical integration for the computation of each of the element stiffness matrices instead of just computing one matrix that holds for all elements in a regular mesh using the standard approach.

For stable convergence the filter is employed using a continuation approach where the value of $\beta$ is gradually increased from 1 to 500 .

The scheme is very similar to the suggested dilate filter operator (18) described in the previous section. Basically, the difference lies in the implementation of the max operator. In the erode scheme, the center pixel is defined as the direct maximum of the neighborhood pixels. In the Heaviside approach, the center pixel is set equal to zero if any of the pixels in the neighborhood is bigger than zero. For a discrete image the two filters are equal but for continuous design variables, the schemes will behave differently.

\section{Modified density filtering with a Heaviside step function}

As was the case for the erosion operator compared to the dilation operator, one may reformulate the idea from Guest et al (2004) discussed above, to turn it into a min-operator based scheme instead of a max-operator based scheme. 
We reformulate the Heaviside operator to give zero density for $\tilde{\rho}_{e}<1$ and only density one for $\tilde{\rho}_{e}=1$, i.e. basically the center pixel only takes the value one if all neighboring elements have density one. The modified Heaviside step function is approximated as a smooth function again governed by the parameter $\beta$

$$
\bar{\rho}_{e}=e^{-\beta\left(1-\tilde{\rho}_{e}\right)}-\left(1-\tilde{\rho}_{e}\right) e^{-\beta} .
$$

For $\beta$ equal to zero, this filter corresponds exactly to (12). For $\beta$ approaching infinity, the Heaviside function is obtained. The structure corresponding to the design variables is in this case much thicker than the physical structure, thus providing more stability during iterations. Also, as will be seen in the examples, this filter works as a "machining" constraint, i.e. the resulting topology has no hole features that are smaller than a circle with radius $R$.

Both the direct Heaviside density filter and the modified version are non-volume preserving. However, in the spirit of the morphology operators, one could combine the two Heaviside filters to produce close and open operators and thereby get volume preservation and other qualities. This idea, however, has not been tested here and will be left for future research.

\subsection{Sensitivity filters}

In the following three modifications of the original sensitivity filter (16) that may be simpler to explain are presented. In general, however, these modified sensitivity filters seem to be less stable as will be seen in the example section.

\section{Alternative sensitivity filter}

In order to obtain a more discrete (black and white) solution than for the original sensitivity filter (16), Borrvall (2001) suggests the following modification

$$
\frac{\widetilde{\partial f}}{\partial \rho_{e}}=\frac{\sum_{i \in N_{e}} w\left(\mathbf{x}_{i}\right) \rho_{i} \frac{\partial f}{\partial \rho_{i}}}{\sum_{i \in N_{e}} \rho_{i} w\left(\mathbf{x}_{i}\right)}
$$

i.e., the density weighting in the denominator is moved inside the summation. The effect of this modification is more black and white solutions at the cost of loss of some mesh-independency effect as will be shown later.

\section{Sensitivity filter without density weighting}

In order to ensure symmetric behavior for multi-phase design problems, the author has previously (Sigmund, 2001b) suggested to drop the density weighting of the original sensitivity filter (16)

$$
\frac{\widetilde{\partial f}}{\partial \rho_{e}}=\frac{\sum_{i \in N_{e}} w\left(\mathbf{x}_{i}\right) \frac{\partial f}{\partial \rho_{i}}}{\sum_{i \in N_{e}} w\left(\mathbf{x}_{i}\right)} .
$$

As for the alternative sensitivity filter above, this modification is introduced at the cost of loss of some of the filtering effect and will thus not be tested for the two-phase problems considered in this paper. 


\section{Mean sensitivity filter}

One may also drop the distance weighting of the filter above and simply modify the sensitivities of element $e$ to become the mean value of the neighboring sensitivities

$$
\frac{\widetilde{\partial f}}{\partial \rho_{e}}=\frac{\sum_{i \in N_{e}} \frac{\partial f}{\partial \rho_{i}}}{\sum_{i \in N_{e}} 1} .
$$

As will be seen in the example section, this filter works surprisingly well in many cases, however, occasionally, especially for more advanced design problems than compliance minimization, it fails in producing checkerboard-free and mesh-independent designs.

\section{Bi-lateral sensitivity filter}

This alternative filter (indirectly ${ }^{2}$ ) proposed in Wang and Wang (2005) is the same as (26), except that the density outside the weighting functions is interchanged with the sensitivity, i.e.

$$
\frac{\widetilde{\partial f}}{\partial \rho_{e}}=\frac{\sum_{i \in N_{e}} w\left(\mathbf{x}_{i}\right) \bar{w}\left(\frac{\partial f}{\partial \rho_{i}}\right) v_{i} \frac{\partial f}{\partial \rho_{i}}}{\sum_{i \in N_{e}} w\left(\mathbf{x}_{i}\right) \bar{w}\left(\frac{\partial f}{\partial \rho_{i}}\right) v_{i}}
$$

where the sensitivity weighting is defined as

$$
\bar{w}\left(\frac{\partial f}{\partial \rho_{i}}\right)=e^{-\frac{1}{2}\left(\frac{\frac{\partial f}{\partial \rho_{i}}-\frac{\partial f}{\partial \rho_{e}}}{\sigma_{r}}\right)^{2}} .
$$

Here the variance factor is $\left.\left.\sigma_{r} \in\right] 0,1\right]$. In contrast to the bi-lateral density filter (26), the author was able to reproduce the examples from Wang and Wang (2005) for the bi-lateral sensitivity filter (33). However, for alternative design problems, such as modelling half the MBB-beam instead of the full beam, the scheme did not result in smooth and mesh-independent results. A two-bar truss problem was also used as a test problem in the paper. Here it was also possible to reproduce the results, even using half the design domain due to symmetry, however, it does not seem justified to prove mesh-convergence for a design problem that is known to have a unique (two-bar) solution.

\section{Implementation of morphology based filters}

Contrary to what one might expect it is not very complicated to implement density based filters in a standard topology optimization software and they may be implemented using black box finite element solvers. Also, the extensions to the morphology-based filters are straightforward.

In order to demonstrate the implementation we first show how the procedure works for the simple original density (12), the dilate (18) and the erode (19) filters.

The sensitivity of an (objective) function $f$ with respect to a change in design variable $\rho_{e}$ is found by use of the chain rule

$$
\frac{\partial f}{\partial \rho_{e}}=\sum_{i \in N_{e}} \frac{\partial f}{\partial \tilde{\rho}_{i}} \frac{\partial \tilde{\rho}_{i}}{\partial \rho_{e}},
$$

\footnotetext{
${ }^{2}$ Many of the examples in the paper are apparently run with this modified filter although the equation is not given explicitly.
} 
where the sensitivity of the filtered density $\tilde{\rho}_{i}$ with respect to a change in design variable $\rho_{e}$ is found as

$$
\frac{\partial \tilde{\rho}_{i}}{\partial \rho_{e}}=\frac{w\left(\mathbf{x}_{e}\right) v_{e}}{\sum_{j \in N_{i}} w\left(\mathbf{x}_{j}\right) v_{j}},
$$

for the original density filter (12). In the case of the dilate operator (18), the sensitivity of the filtered density becomes

$$
\frac{\partial \tilde{\rho}_{i}}{\partial \rho_{e}}=\frac{e^{\beta \rho_{e}}}{\sum_{j \in N_{i}} e^{\beta \rho_{j}}} .
$$

The sensitivity expression for the close operator is similar to (37) with $\rho_{k}$ interchanged with $1-\rho_{k}$. The sensitivity expressions for the open and close operators become a bit more involved and are discussed later.

The main computational burden of the simple filtering schemes (the original density filter and the dilate and erode operators) is to find the neighbors to each element. This is especially true in the case of irregular meshes. Therefore, we suggest to compute a "neighborhood" matrix $\mathbf{N}$ that contains rows with neighbors to each element in the structure once and for all before the optimization begins. Following this idea, a very simplified flow chart in pseudo code may look like

1. Build neighborhood matrix $\mathbf{N}$

2. Initialize design variable vector $\boldsymbol{\rho}$, counter iter $=0$ and change $=1$

3. while change $>0.01$ and iter $\leq 1000$

4. $\quad$ iter $=$ iter +1

5. Compute filtered densities $\tilde{\boldsymbol{\rho}}$ using (12) (or (18) or (19))

6. Solve FE problem based on filtered sensitivities $\tilde{\boldsymbol{\rho}}$

7. Calculate intermediate sensitivities based on filtered densities, i.e.

$$
\frac{\partial f}{\partial \tilde{\rho}_{e}}=-\mathbf{u}_{e}^{T} \frac{\partial \mathbf{k}_{e}}{\partial \tilde{\rho}_{e}} \mathbf{u}_{e}-p \tilde{\rho}_{e}^{p-1} \mathbf{u}_{e}^{T} \mathbf{k}_{\mathbf{e}}^{0} \mathbf{u}_{\mathbf{e}}
$$

8. Compute final sensitivities from (35) using (36)

9. Update design variables $\boldsymbol{\rho}_{\text {new }}$ using MMA

10. Calculate change $=\left\|\boldsymbol{\rho}_{\text {new }}-\boldsymbol{\rho}\right\|_{\infty}$

11. end

For the filters involving $\beta$ continuation schemes, the value of $\beta$ is initialized in step 2 and step 9 is substituted with

9. if $\left\{\bmod (\right.$ iter, 50$)=1$ ' or 'change $<0.01$ ' and ' $\beta \leq \beta_{\text {max }}$ ' $\}$ then

$$
\begin{aligned}
& \beta=2 \beta \\
& \text { change }=0.5
\end{aligned}
$$

end 
where $\beta_{\max }$ is the maximum value of $\beta$ which is 200 for the morphology schemes and 500 for the Heaviside schemes.

Only general guidelines for the sensitivity analysis for the more complex morphology operators will be given in the following. For the simple erode and dilate schemes, the sensitivity of the objective function can be written as equation (35) with (37), as discussed above. For the close (combined dilate and erode) operator it expands to

$$
\frac{\partial f}{\partial \rho_{e}}=\sum_{i \in N_{e}} \sum_{j \in N_{i}} \frac{\partial f}{\partial \tilde{\tilde{\rho}}_{j}} \frac{\partial \overline{\tilde{\rho}}_{j}}{\partial \tilde{\rho}_{i}} \frac{\partial \tilde{\rho}_{i}}{\partial \rho_{e}} .
$$

The sensitivity of the open operator is obtained by switching tilde and bars. For the close-open operator, the sensitivity expression expands to

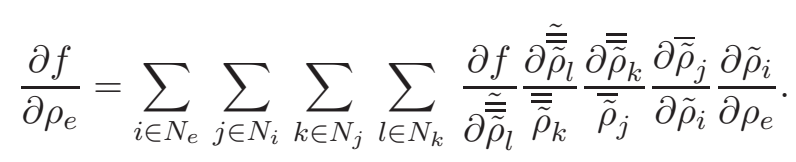

It is seen that the computational burden is growing immensely for the combined open-close and close-open schemes. Assuming a design domain discretized by a thousand elements, a neighborhood comprising 9 elements and ignoring boundary effects, the number of filter summation operations for the simple erode or dilate filters is 9,000, for the open and close filters it is 81,000 and for the openclose and close-open filters it is 6,561,000. The general expressions for the number of summation operations for the three cases is $\operatorname{size}\left(N_{e}\right) n, \operatorname{size}\left(N_{e}\right)^{2} n$ and $\operatorname{size}\left(N_{e}\right)^{4} n$, respectively, where $n$ is the number of design elements and $N_{e}$ is the neighborhood of element $e$. For a more realistic (but still rather academic) problem like the MBB test example considered in this paper, size $\left(\mathrm{N}_{\mathrm{e}}\right)=37$ and $n=4800$. This results in $9 \times 10^{9}$ summation operations. This number should be compared to an estimate of $b w^{2} \times n_{d o f}$ multiplication for the decomposition of a symmetric band matrix, where $b w$ is the bandwidth of the stiffness matrix and $n_{\text {dof }}$ is the number of degrees of freedom. In the MBB test example this corresponds to approximately $70 \times 10^{6}$ multiplication, i.e. a factor of at least 100 times less than for the filter operation. In conclusion, the open-close and close-open filters are not practically applicable in large scale problems. However, as will be demonstrated in the example section it is plausible to use the combined filter (24) (with a summation count of $6 \times 10^{6}$ for the MBB-example) until convergence followed by a few iterations with the full open-close or close-open filters to obtain reasonable designs with minimum feature size control for both holes and structural details.

\section{Test of the filters}

It is difficult to make a fair comparison of the different filter methods. For sure, the efficiency of each filter has been optimized by the respective authors and fine-tuning of involved parameters may be important. In the following, we test the different filters with standard parameters for the three test problems. The main aim is to test and compare the different filters for their stability and efficiency for practical applications. If a filter technique requires too much fine-tuning of parameters to work well on a specific example, it will anyway not be worthwhile using it in practice.

All the filters have been implemented in a MATLAB setting and are solved using MMA (Svanberg, 1987) with standard settings. Each test example is run using 15 different filtering schemes and the results are presented in figures and tables. As discussed earlier, we plot both the non-physical design variable distribution $\boldsymbol{\rho}$ and the physical density distribution $\tilde{\boldsymbol{\rho}}$ (the densities used in the SIMP interpolation scheme (10)) in all figures. For the physical interpretation of a design, it is obviously the latter plot that must be consulted. In order to have a "measure of discreteness", i.e. a way to tell 


\begin{tabular}{|l|l|l|r|r|r|r|}
\hline Filter type & Eq. & Fig. & $f$ & $V / V^{*}$ & $M_{n d}(\%)$ & It. \\
\hline \hline No filtering & - & $4 \mathrm{a}$ & 196.8 & 0.5 & 1.45 & 50 \\
\hline \hline \multicolumn{7}{|l|}{ Standard filters } \\
\hline \hline Density w. lin. weight & $(12),(13)$ & $4 \mathrm{~b}$ & 218.3 & 0.5 & 24.02 & 1000 \\
\hline Bi-lateral density & $(26)$ & $4 \mathrm{c}$ & 213.6 & 0.5 & 21.92 & 73 \\
\hline Heaviside & $(28)$ & $4 \mathrm{~d}$ & 190.0 & 0.5 & 0.36 & 489 \\
\hline Modified Heaviside & $(29)$ & $4 \mathrm{e}$ & 192.3 & 0.5 & 0.23 & 435 \\
\hline Sensitivity & $(17)$ & $4 \mathrm{f}$ & 210.0 & 0.5 & 20.41 & 119 \\
\hline Mean sensitivity & $(32)$ & $4 \mathrm{~g}$ & 192.5 & 0.5 & 0.48 & 1000 \\
\hline Bi-lateral sensitivity & $(33)$ & $4 \mathrm{~h}$ & 186.6 & 0.5 & 0.89 & 225 \\
\hline \hline New morphology-based filters & \multicolumn{5}{|c|}{} \\
\hline \hline Dilate & $(18)$ & $5 \mathrm{a}$ & 192.9 & 0.5 & 2.24 & 532 \\
\hline Erode & $(19)$ & $5 \mathrm{~b}$ & 197.3 & 0.5 & 1.42 & 474 \\
\hline Close & $(20)$ & $5 \mathrm{c}$ & 195.4 & 0.5 & 1.19 & 414 \\
\hline Open & $(21)$ & $5 \mathrm{~d}$ & 192.9 & 0.5 & 1.84 & 423 \\
\hline Combi & $(24)$ & $5 \mathrm{e}$ & 194.6 & 0.5 & 1.71 & 425 \\
\hline Close-Open & $(22)$ & $5 \mathrm{f}$ & 196.2 & 0.5 & 2.31 & $(444)$ \\
\hline Open-Close & $(23)$ & $5 \mathrm{~g}$ & 196.2 & 0.5 & 2.50 & $(439)$ \\
\hline \hline
\end{tabular}

Table 1: Results for the MBB example.

whether an optimized design has converged to a discrete solution, we introduce the measure

$$
M_{n d}=\frac{\sum_{e=1}^{n} 4 \tilde{\rho}_{e}\left(1-\tilde{\rho}_{e}\right)}{n} \times 100 \% .
$$

If a converged design is fully discrete, i.e. there are no elements with intermediate density values, $M_{n d}$ is $0(\%)$. If the design is totally grey, i.e. all elements densities are equal to $0.5, M_{n d}$ is $100(\%)$. Hence, the measure may be called a Measure of Non-Discreteness $\left(M_{n d}\right)$.

In the following, we discuss the results for the three different test cases.

\subsection{The MBB test example}

The results for the MBB test example are shown in Figs. 4 and 5 and the data for the results are given in Tab. 1.

An examination of Figs. 4 and 5 reveals that the resulting topologies may be very different for the various filters even though the filter radius $R$ is the same. However, the cases which do not correspond to the simple MBB topology in Fig. $4 \mathrm{~b}$ are the cases where the filter radius is not directly connected with topological features. If our goal is to select methods that impose minimum length-scales and manufacturability, we can at this point already dispose of the two Bi-lateral filter methods (Figs. 4c and $\mathrm{h}$ ) and possibly the mean sensitivity filter (Fig. 4g). The rest of the topologies are very similar with the exceptions of the original density filter result (Fig. 4b) and the sensitivity filter result (Fig. 4f) which (as expected) show grey transitions between the solid and void regions. These filter-enforced grey regions are also reflected in the obtained compliances that are approximately $10 \%$ higher than for the discrete designs.

The results for the Heaviside (Fig. 4d), dilate (Fig. 5a) and open (Fig. 5d) filters are very similar. It is seen that the filters correspond to deposition tools, i.e. there are no structural details below the 
- Filter size
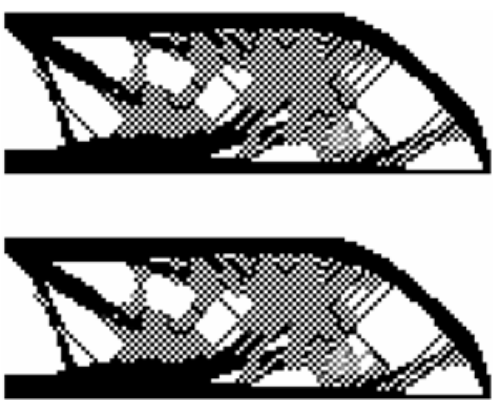

a) No filtering
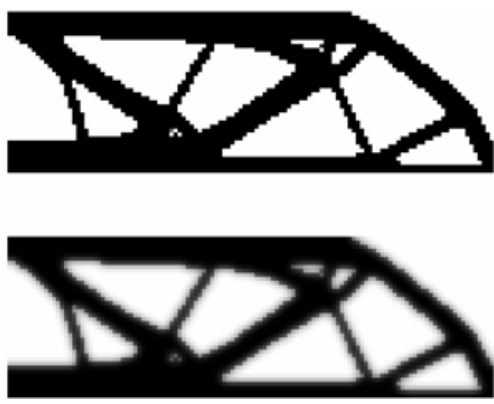

c) Bi-lateral density
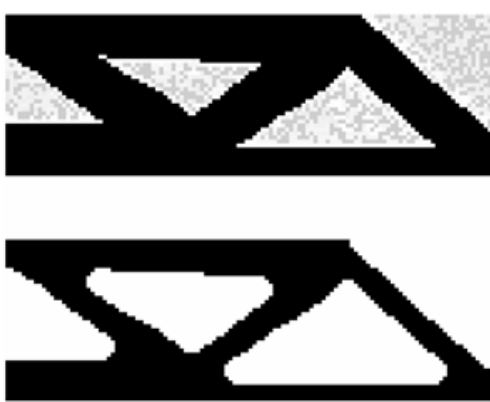

e) Modified Heaviside
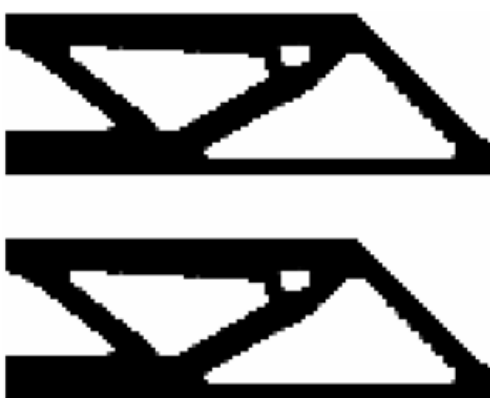

g) Mean sensitivity
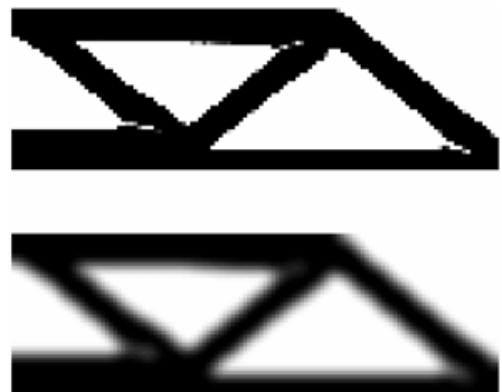

b) Density w. lin. weight
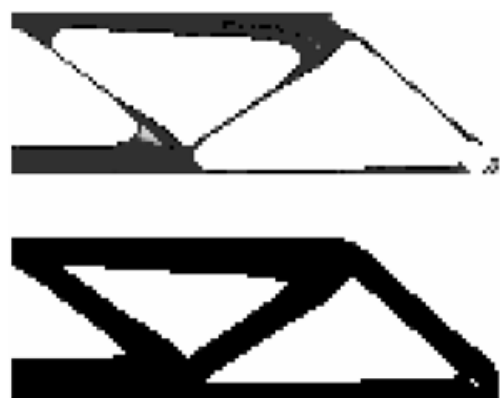

d) Heaviside
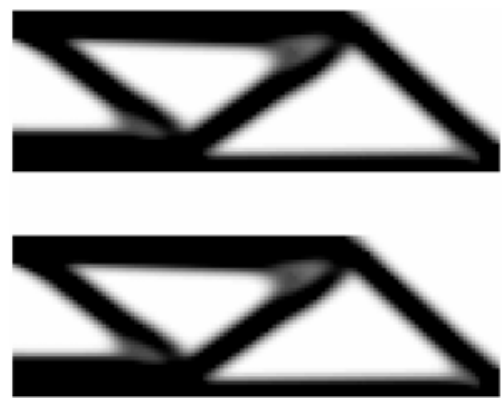

f) Sensitivity
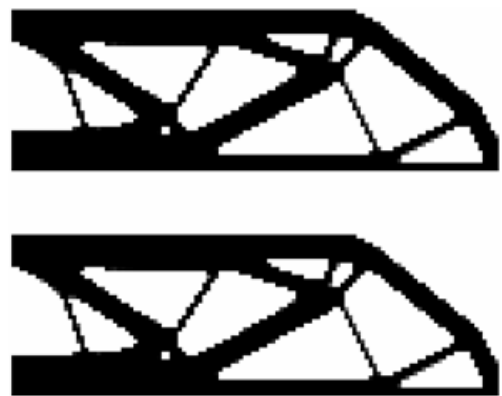

h) Bi-lateral sensitivity

Figure 4: Results for the MBB test example with standard filters. For each filter, two images are shown. The upper one shows the (non-physical) design variable field $\boldsymbol{\rho}$ and the lower one shows the filtered (physical) density field $\tilde{\boldsymbol{\rho}}$. a) No filtering, b) Density filter with linearly decaying weighting (12), (13), c) Bi-lateral density filter (26), d) Density filter with a Heaviside step function (28), e) Modified density filter with a Heaviside step function (29), f) Original sensitivity filter (17), g) Mean sensitivity filter (32) and h) Bi-lateral sensitivity filter (33). 
Filter size
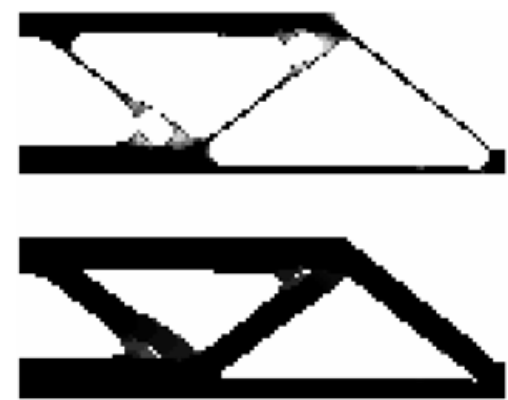

a) Dilate
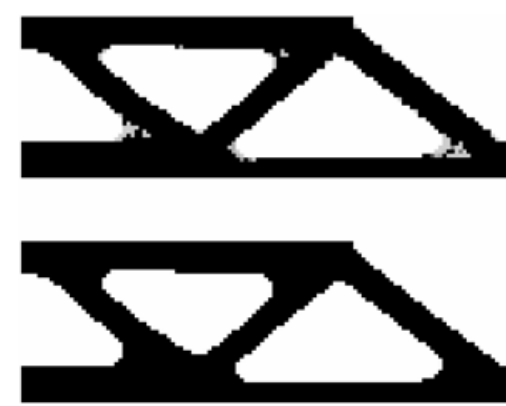

c) Close
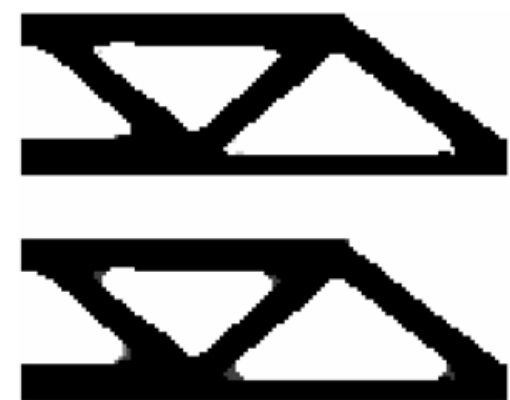

e) Combi
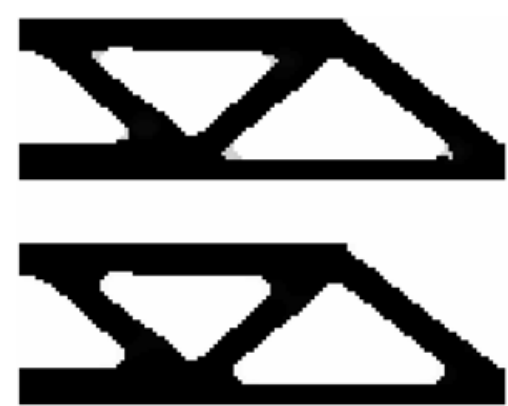

g) Open-Close
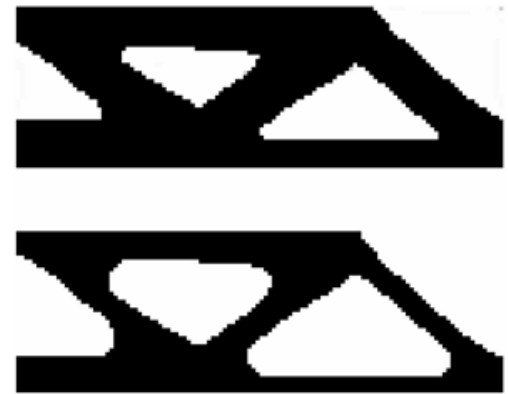

b) Erode
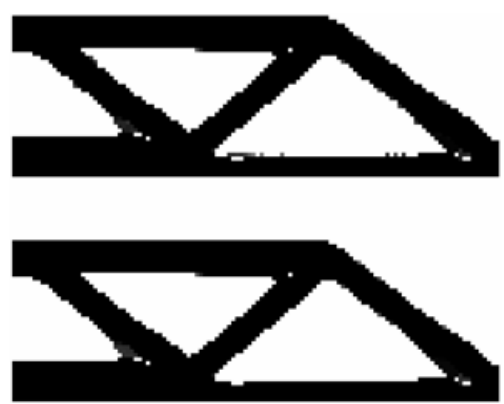

d) Open
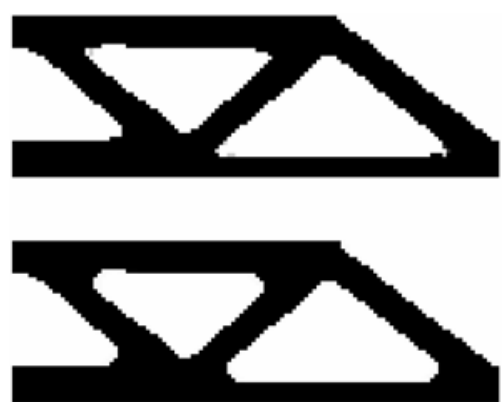

f) Close-Open

igure 5: Results for the MBB test example with new morphology based filters. For each filter, two images are shown. The upper one shows the (non-physical) design variable field $\boldsymbol{\rho}$ and the lower one shows the filtered (physical) density field $\tilde{\boldsymbol{\rho}}$. a) Dilate filter (18), b) Erode filter (19), c) Close filter (20), d) Open (21), e) Combination of the Close and Open filters (24), f) Close-Open filter (22) and g) Open-Close filter (23). 


\begin{tabular}{|l|l|l|r|r|r|r|}
\hline Filter type & Eq. & Fig. & $f$ & $V / V^{*}$ & $M_{n d}(\%)$ & It. \\
\hline \hline No filtering & - & $6 \mathrm{a}$ & -1.33 & 0.25 & 1.43 & 57 \\
\hline \hline Standard filters & \multicolumn{7}{|l|}{} \\
\hline \hline Density w. lin. weight & $(12),(13)$ & $6 \mathrm{~b}$ & -2.05 & 0.25 & 15.18 & 463 \\
\hline Bi-lateral density & $(26)$ & $6 \mathrm{c}$ & -2.03 & 0.25 & 8.46 & 182 \\
\hline Heaviside & $(28)$ & $6 \mathrm{~d}$ & -2.34 & 0.25 & 0.35 & 552 \\
\hline Modified Heaviside & $(29)$ & $6 \mathrm{e}$ & -2.31 & 0.25 & 0.04 & 467 \\
\hline Sensitivity & $(17)$ & $6 \mathrm{f}$ & -2.20 & 0.25 & 12.00 & 844 \\
\hline Mean sensitivity & $(32)$ & $6 \mathrm{~g}$ & -2.27 & 0.25 & 0.63 & 1000 \\
\hline Bi-lateral sensitivity & $(33)$ & $6 \mathrm{~h}$ & -2.16 & 0.25 & 0.68 & 410 \\
\hline \hline New morphology-based filters & & \multicolumn{5}{|c|}{} \\
\hline \hline Dilate & $(18)$ & $7 \mathrm{a}$ & -2.26 & 0.25 & 0.90 & 624 \\
\hline Erode & $(19)$ & $7 \mathrm{~b}$ & -2.27 & 0.25 & 1.08 & 503 \\
\hline Close & $(20)$ & $7 \mathrm{c}$ & -2.22 & 0.25 & 2.35 & 378 \\
\hline Open & $(21)$ & $7 \mathrm{~d}$ & -2.24 & 0.25 & 0.88 & 468 \\
\hline Combi & $(24)$ & $7 \mathrm{e}$ & -2.19 & 0.25 & 1.03 & 525 \\
\hline Close-Open & $(22)$ & $7 \mathrm{f}$ & -2.18 & 0.25 & 2.00 & $(538)$ \\
\hline Open-Close & $(23)$ & $7 \mathrm{~g}$ & -2.23 & 0.25 & 1.67 & $(571)$ \\
\hline \hline
\end{tabular}

Table 2: Results for the inverter example.

size of the filter, however, nothing prevents small crack-like air holes from being formed. Although these holes will be diminished with mesh refinement, sharp re-entrant corners also form that may be unwanted for stress reasons. As an alternative, the modified Heaviside (Fig. 4e), erode (Fig. 5b) and close (Fig. 5c) filters all provide topologies with smoothed corners no sharper than allowed by the filter radius. In principle, the latter filters allow smaller structural details than the filter size but such features are not seen in this example. It is also noted how the latter filters work as machining tools, i.e. the topologies could all be machined with a tool of the size of the filter. In order to ensure both minimum hole and minimum structural feature sizes, the combi, close-open and open-close filters (Figs. 5e-g) could do the job, but again, this is not demonstrated with the MBB example.

Examining Tab. 1 it is noted that the lowest compliances are obtained for the bi-lateral sensitivity filter but this is obtained on the cost of mesh-dependence. Otherwise, the compliances are fairly constant with the exception of the original sensitivity and density filters. It is noted that the Heaviside filters seem to be slightly better in compliance and discreteness than their morphological counterparts. This may partly be attributed to the higher $\beta$ value and partly because there is a built-in artificial penalization in the Heaviside filter. This postulate is supported with a small example. Assuming that the neighborhood comprises three elements with the values $0.2,1$ and 0.2 , respectively, then the modified Heaviside filter will result in a filtered center density that approaches 0 as $\beta$ approaches infinity. For the corresponding erode operator, the filtered center density will approach 0.2 as $\beta$ approaches infinity (i.e. it is a true min-operator). The results of this difference will be more clearly seen in the wave transmitter example.

\subsection{The inverter test example}

The results for the inverter test example are shown in Figs. 6 and 7 and the data for the results are given in Tab. 2.

Most of the conclusions drawn for the MBB example also apply for the inverter example but some weak points of the different filter schemes are exhibited even more clearly in the hinge regions of the resulting 
- Filter size
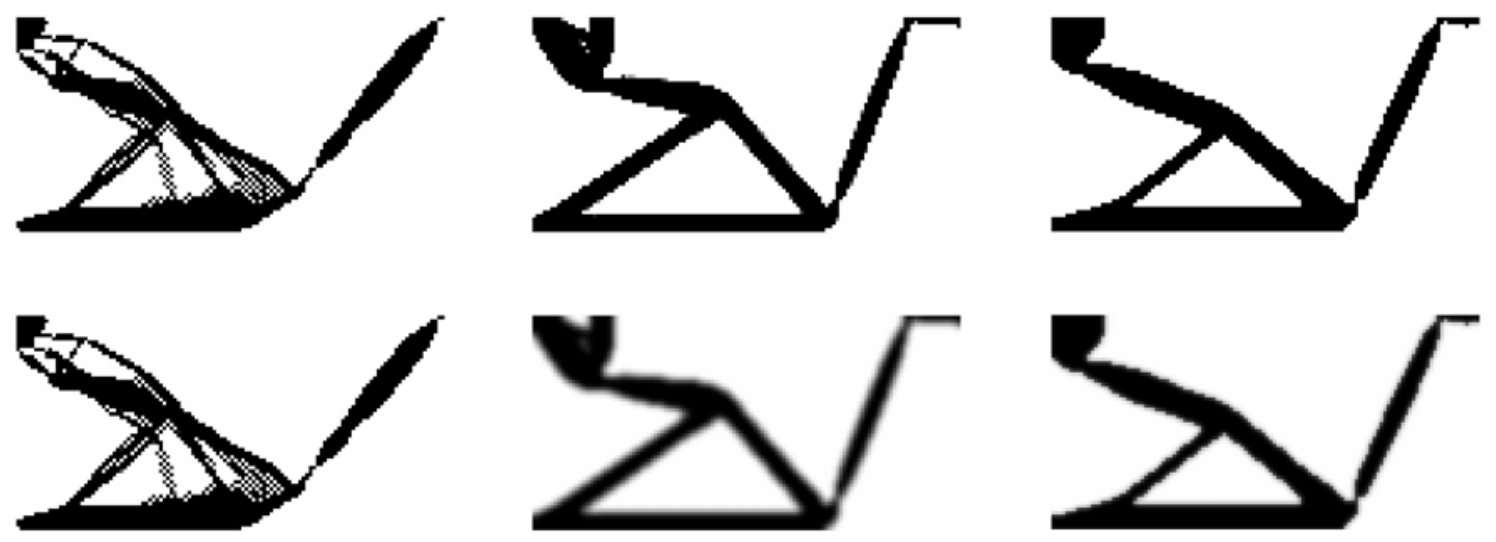

a) No filtering

b) Density w. lin. weight

c) Bi-lateral density
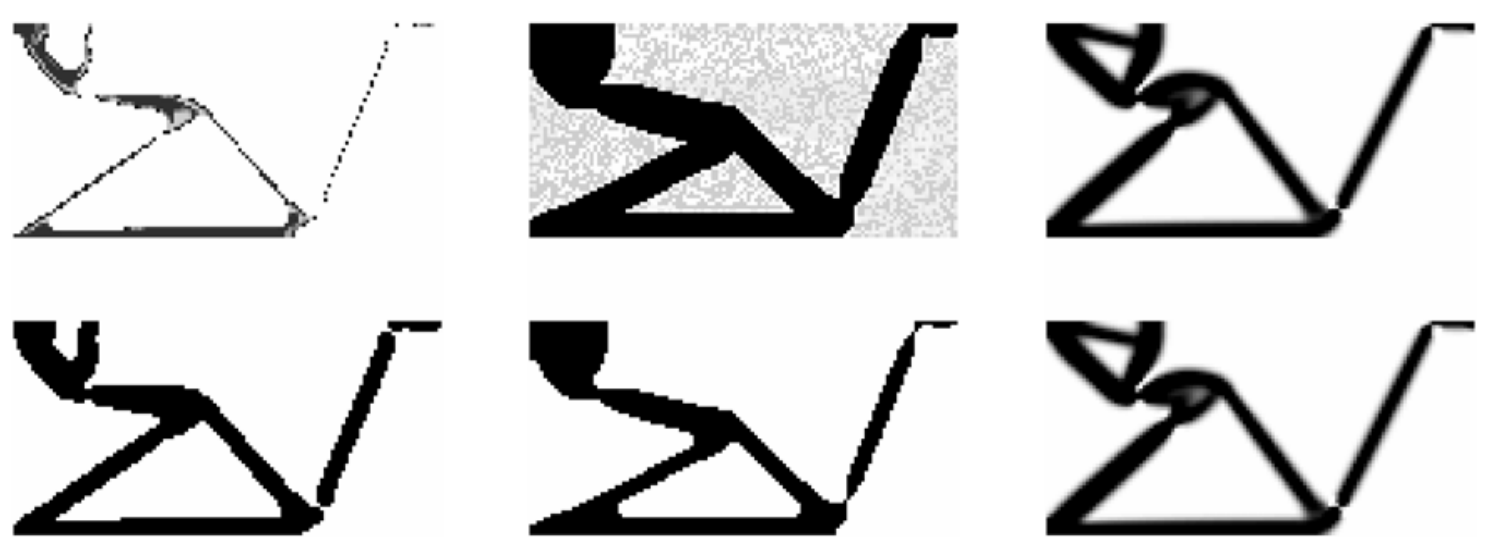

d) Heaviside

e) Modified Heaviside

f) Sensitivity
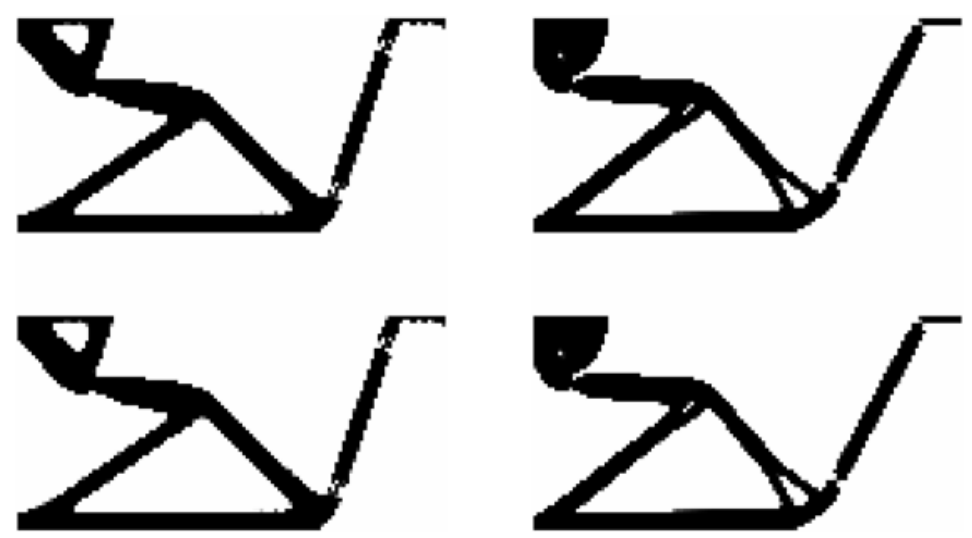

g) Mean sensitivity

h) Bi-lateral sensitivity

Figure 6: Results for the inverter test example with standard filters. For each filter, two images are shown. The upper one shows the (non-physical) design variable field $\boldsymbol{\rho}$ and the lower one shows the filtered (physical) density field $\tilde{\boldsymbol{\rho}}$. a) No filtering, b) Density filter with linearly decaying weighting (12), (13), c) Bi-lateral density filter (26), d) Density filter with a Heaviside step function (28), e) Modified density filter with a Heaviside step function (29), f) Original sensitivity filter (17), g) Mean sensitivity filter (32) and h) Bi-lateral sensitivity filter (33). 
- Filter size
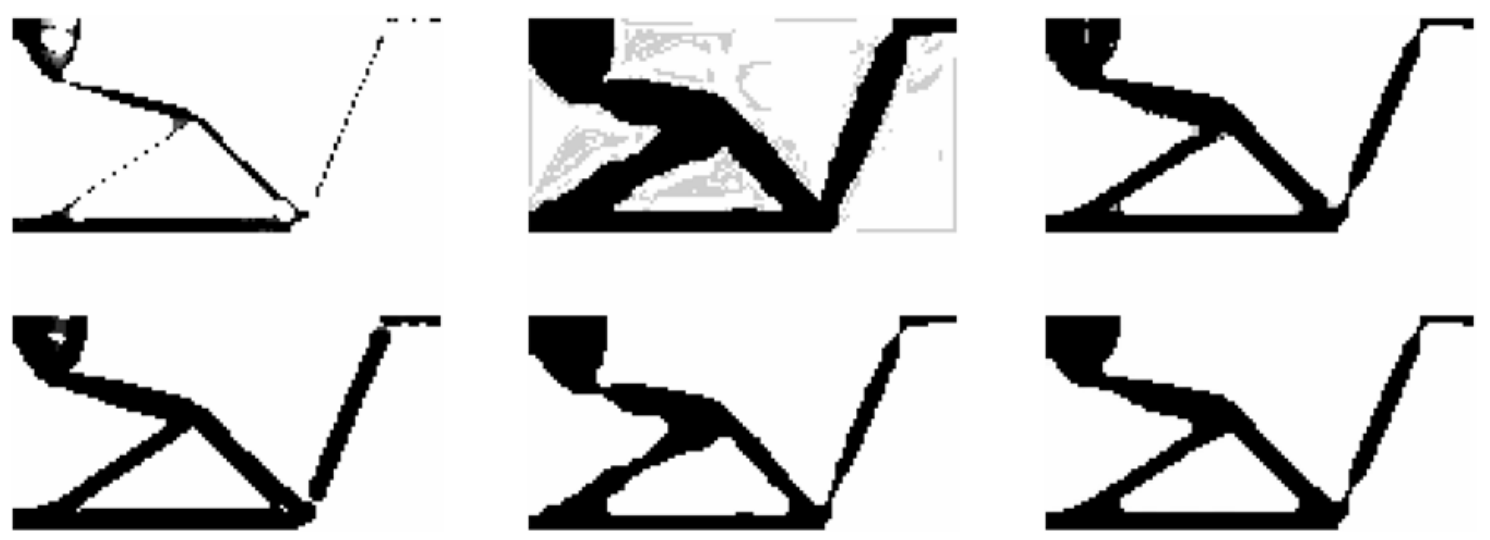

a) Dilate

b) Erode

c) Close
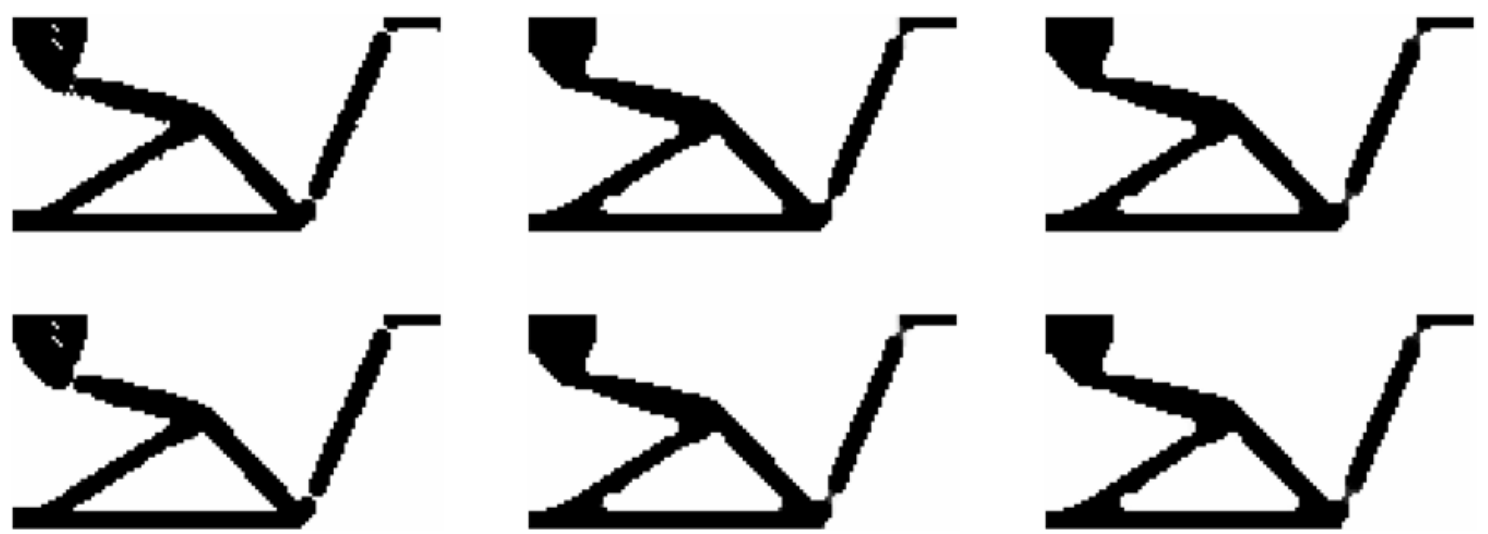

d) Open

e) Combi

f) Close-Open
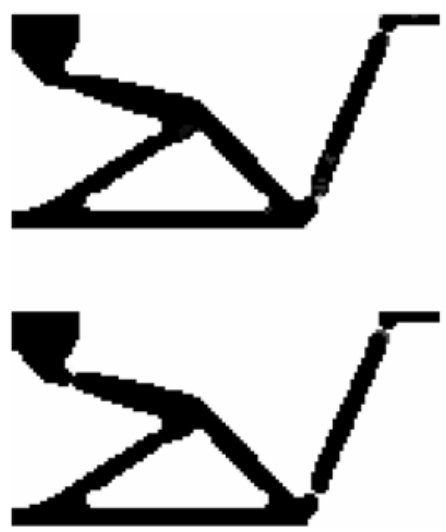

g) Open-Close

Figure 7: Results for the inverter test example with new morphology based filters. For each filter, two images are shown. The upper one shows the (non-physical) design variable field $\boldsymbol{\rho}$ and the lower one shows the filtered (physical) density field $\tilde{\boldsymbol{\rho}}$. a) Dilate filter (18), b) Erode filter (19), c) Close filter (20), d) Open (21), e) Combination of the Close and Open filters (24), f) Close-Open filter (22) and g) Open-Close filter (23). 


\begin{tabular}{|l|l|l|r|r|r|r|}
\hline Filter type & Eq. & Fig. & $f$ & $V / V^{*}$ & $M_{n d}(\%)$ & It. \\
\hline \hline No filtering & - & $8 \mathrm{a}$ & -5.78 & 0.53 & 16.25 & 1000 \\
\hline \hline \multicolumn{7}{|l|}{} \\
\hline Standard filters & \multicolumn{7}{|l|}{} \\
\hline \hline Density w. lin. weight & $(12),(13)$ & $8 \mathrm{~b}$ & -5.42 & 0.71 & 22.67 & 566 \\
\hline Bi-lateral density & $(26)$ & $8 \mathrm{c}$ & -5.57 & 0.70 & 19.19 & 539 \\
\hline Heaviside & $(28)$ & $8 \mathrm{~d}$ & -6.55 & 0.90 & 0.87 & 563 \\
\hline Modified Heaviside & $(29)$ & $8 \mathrm{e}$ & -6.45 & 0.28 & 1.06 & 856 \\
\hline Sensitivity & $(17)$ & $8 \mathrm{f}$ & -6.32 & 0.90 & 4.81 & 929 \\
\hline Mean sensitivity & $(32)$ & $8 \mathrm{~g}$ & -6.37 & 0.81 & 3.45 & 635 \\
\hline Bi-lateral sensitivity & $(33)$ & $8 \mathrm{~h}$ & -6.31 & 0.63 & 10.29 & 643 \\
\hline \hline New morphology-based filters & & \multicolumn{5}{|c|}{} \\
\hline \hline Dilate & $(18)$ & $9 \mathrm{a}$ & -5.86 & 0.88 & 2.75 & 694 \\
\hline Erode & $(19)$ & $9 \mathrm{~b}$ & -5.18 & 0.63 & 4.96 & 867 \\
\hline Close & $(20)$ & $9 \mathrm{c}$ & -4.97 & 0.79 & 5.30 & 652 \\
\hline Open & $(21)$ & $9 \mathrm{~d}$ & -5.31 & 0.83 & 1.87 & 606 \\
\hline Combi & $(24)$ & $9 \mathrm{e}$ & -5.25 & 0.80 & 4.46 & 839 \\
\hline Close-Open & $(22)$ & $9 \mathrm{f}$ & -5.09 & 0.81 & 5.81 & $(1147)$ \\
\hline Open-Close & $(23)$ & $9 \mathrm{~g}$ & -4.84 & 0.86 & 4.53 & $(1164)$ \\
\hline \hline
\end{tabular}

Table 3: Results for the wave transmitter test example.

inverter topologies. As mentioned in Section 2, a weak point of the topology optimization method in its application to compliant mechanism design is the modelling and design of hinges. Basically, the optimizer wants to make optimal but non-physical one-node connected hinges that can transfer forces without bending resistance. The problem is mostly due to modelling issues. In reality, infinite stresses would appear in the hinges which would then fail. In order to prevent this, stresses in the hinge regions should be modelled correctly with higher order elements and mesh-refinement and stress constraints should be imposed. However, this is impractical and may not resolve the problem entirely. Ideally a filter should take care of the problem but as is seen in Figs. 6 and 7 most of the filters do not. The only filter schemes that result in slender non-corner-connected hinges and discrete results are the modified Heaviside, close, combi and close-open filters (Figs. 6e, 7c, e and f). In principle, the dilate, open, open-close and Heaviside filters should also prevent the hinges with mesh-refinement, however, due to the staircase nature of the neighborhood region, it is often possible for the optimizer to find topologies where two circle like regions are only touching in one corner, resulting in a one-node connected hinge. Defining the design variables in the nodal points (as in Guest et al (2004)) may relieve the problem although it has not been tested.

Judging from the objective function in Tab. 2 it is clear that all the discrete filters perform almost equally well with the hinge-preventing schemes (close, close-open and combi) being a bit worse since the distributed hinges are structurally non-efficient (with the finite element model applied). Again, the Heaviside filter result in more discrete solutions for the reasons discussed under the MBB example.

\subsection{The wave transmitter test example}

The results for the wave transmitter test example are shown in Figs. 8 and 9 and the data for the results are given in Tab. 3 .

Due to the non-existing volume constraint and the special nature of wave propagation problems, the transmitter example really exhibits the differences between filters. Examining the result plots in Figs. 8 and 9 and Tab. 3 it is clear that topologically extremely different structures may have very similar 
- Filter size
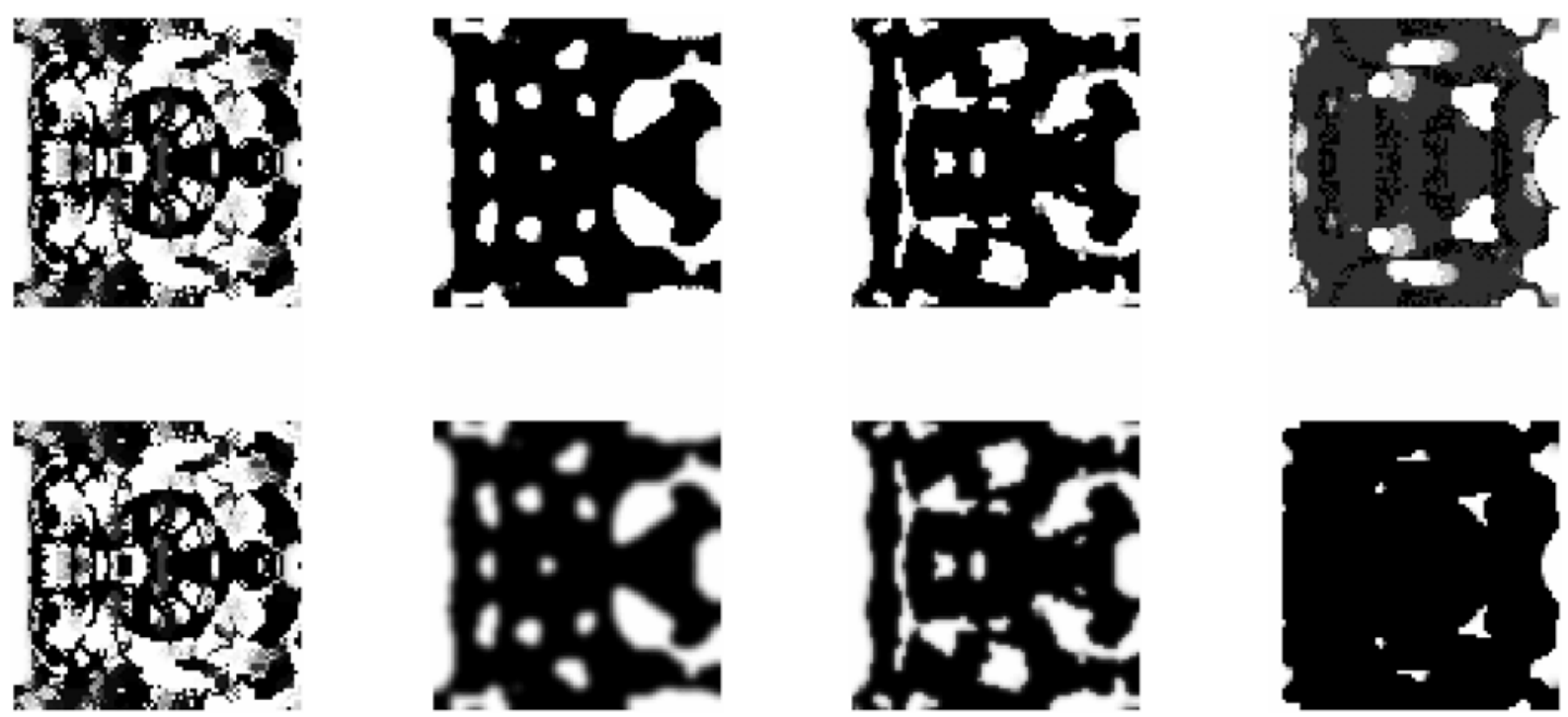

a) No filtering

b) Density w. const. weight c) Bi-lateral density

d) Heaviside
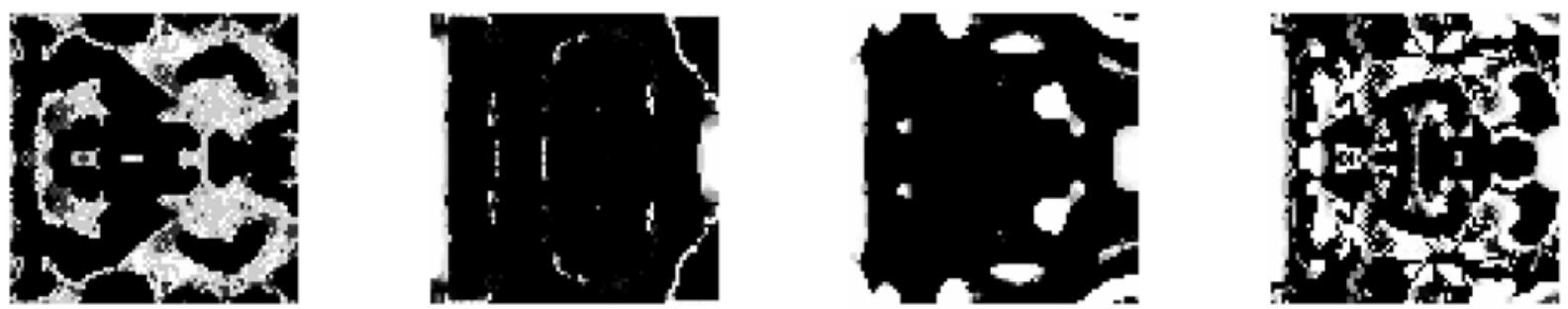

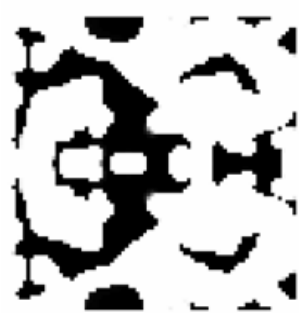

e) Modified Heaviside

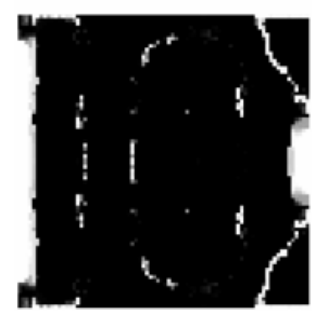

f) Sensitivity

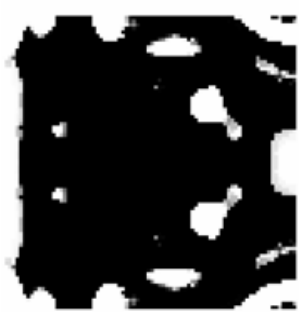

g) Mean sensitivity

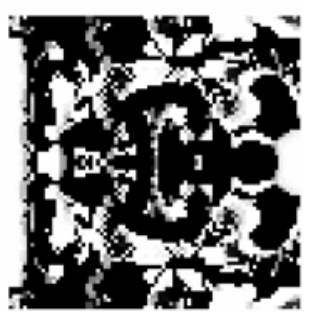

h) Bi-lateral sensitivity

Figure 8: Results for the wave transmitter test example with standard filters. For each filter, two images are shown. The upper one shows the (non-physical) design variable field $\boldsymbol{\rho}$ and the lower one shows the filtered (physical) density field $\tilde{\boldsymbol{\rho}}$. a) No filtering, b) Density filter with linearly decaying weighting (12), (13), c) Bi-lateral density filter (26), d) Density filter with a Heaviside step function (28), e) Modified density filter with a Heaviside step function (29), f) Original sensitivity filter (17),

g) Mean sensitivity filter (32) and h) Bi-lateral sensitivity filter (33). 
- Filter size
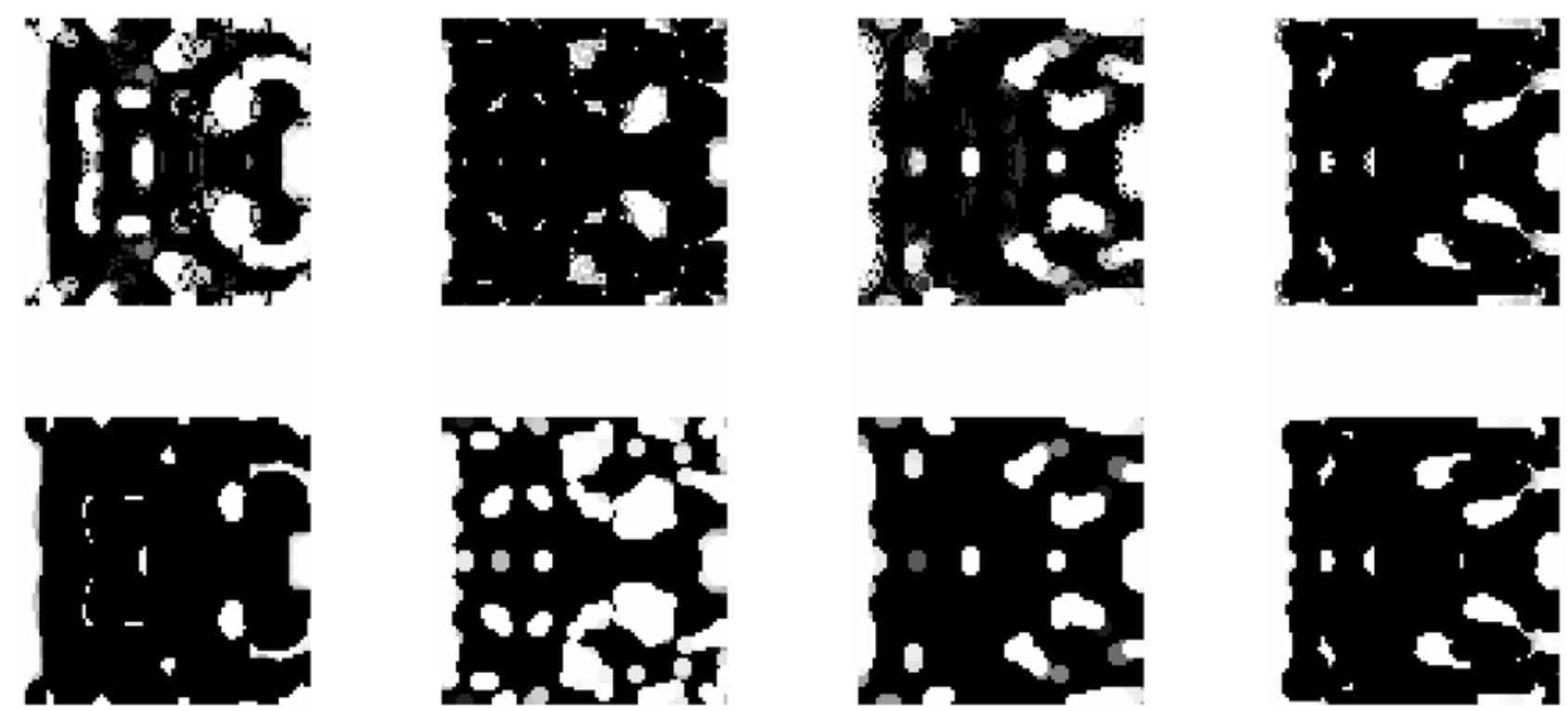

a) Dilate

b) Erode

c) Close

d) Open
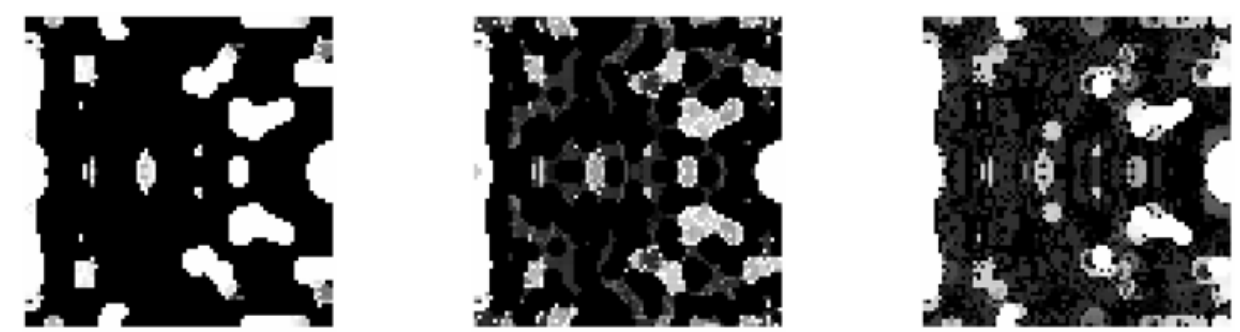

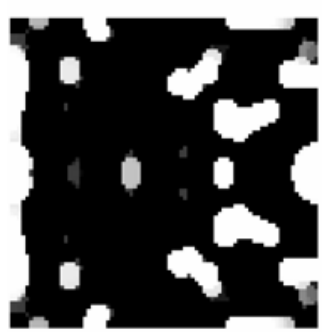

e) Combi

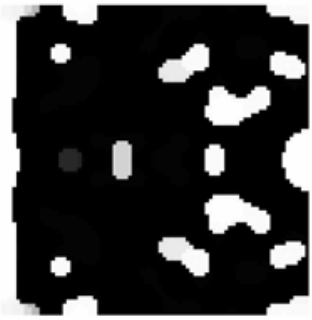

f) Close-Open

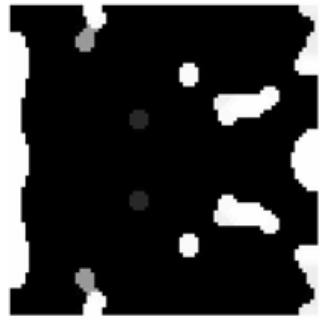

g) Open-Close

Figure 9: Results for the wave transmitter test example with new morphology based filters. For each filter, two images are shown. The upper one shows the (non-physical) design variable field $\boldsymbol{\rho}$ and the lower one shows the filtered (physical) density field $\tilde{\boldsymbol{\rho}}$. a) Dilate filter (18), b) Erode filter (19), c) Close filter (20), d) Open (21), e) Combination of the Close and Open filters (24), f) Close-Open filter (22) and g) Open-Close filter (23). 
objective function values which means that we are dealing with an optimization problem with a highly non-unique solution. It is also clear that filtering is absolutely essential for getting any structurally interpretable design.

For the black and white filters, the results can be divided into the dilate-type filters with minimum structural detail size but very small hole (epoxy) features, i.e. the Heaviside (Fig. 8d), dilate (Fig. 9a) and open (Fig. 9d) filters; the erode-type filters with minimum hole sizes, i.e. the modified Heaviside (Fig. 8e), erode (Fig. 9b) and close (Fig. 9c) filters; and the filters that take care of both minimum hole and structural sizes, i.e. the combi (Fig. 9e), close-open (Fig. 9f) and open-close (Fig. 9g). Again, the Heaviside filters result in the best objective function values. Their superiority in providing discrete designs is more clear in the present case where the morphology filters exhibit some problems with intermediate density features. Although this problem could be resolved by raising the pamping parameter, the Heaviside filters are to be preferred here, since they have a "built-in" discreteness which diminishes the need for the penalization and/or pamping of intermediate densities.

\subsection{Summary of examples}

The only filters that have worked well for all examples are the new morphology and the Heaviside filters. In all cases, the Heaviside filters have provided the best and most discrete designs, tightly followed by the morphology based schemes. The alternative black/white filters like the two bilateral filters, and the mean sensitivity filters may in many cases provide nice black and white solutions, but there is no enforcement of a strict length-scale, hence they cannot be recommended as general purpose filters.

In many cases it is fine to work with the non-volume preserving filters such as the Heaviside filters, and the dilate and erode filters. However, if there are fixed solid or void regions involved in the design problems which was not demonstrated in the three test cases, it is preferable to work with volume preserving filters (such as the open or close filters). The reason is that details with features smaller than the neighborhood area may appear at boundaries adjacent to the passive regions as illustrated in Fig. 10.

In conclusion, we recommend the open and close filters as stable and general filters which still are computationally efficient. The sensitivity analyses for the open-close and close-open operators are very CPU-time consuming and cannot be recommended for general use. However, the combined scheme (24) composed as the average of the open and close operators constitutes a satisfactory replacement.

The reason for not directly recommending the Heaviside schemes is that they are not volume preserving and hence fail to provide length-scale constraints for the fixed solid/void domain case as discussed above. However, it should be plausible to combine the Heaviside schemes into "Heaviside close and open" morphological schemes just as done for the open and close operators. Although not tested here, the author believes that this idea will be rewarding with respect to computational stability and the enforcement of black and white solutions. This will also avoid the need for the special precautions in the $\beta$-continuation approach discussed in Guest et al (2004), since the filters now become volume preserving.

Finally, it should be mentioned that all the proposed filters can be applied to nodal-based design variables as well. In fact this has been tested by the author for the MBB example with the same conclusions as above and the results have therefore been omitted from the paper.

\section{Conclusions}

This paper has suggested a new family of morphology based density filtering schemes for topology optimization that provides mesh-independent, discrete and manufacturable solutions. The methods 
a)
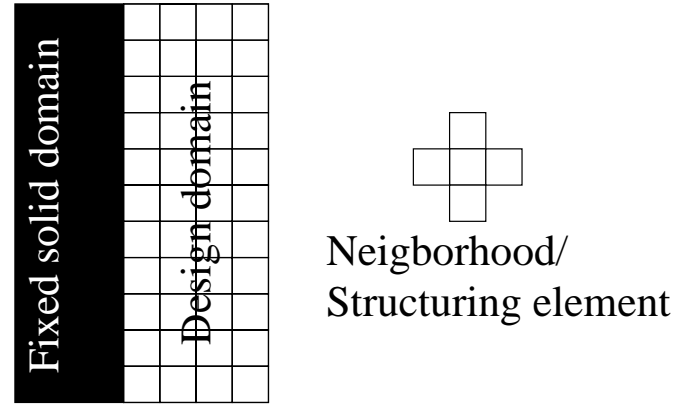

b)
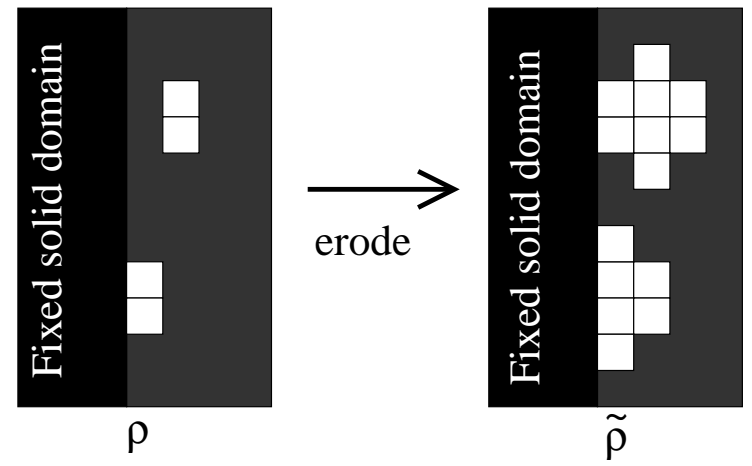

c)
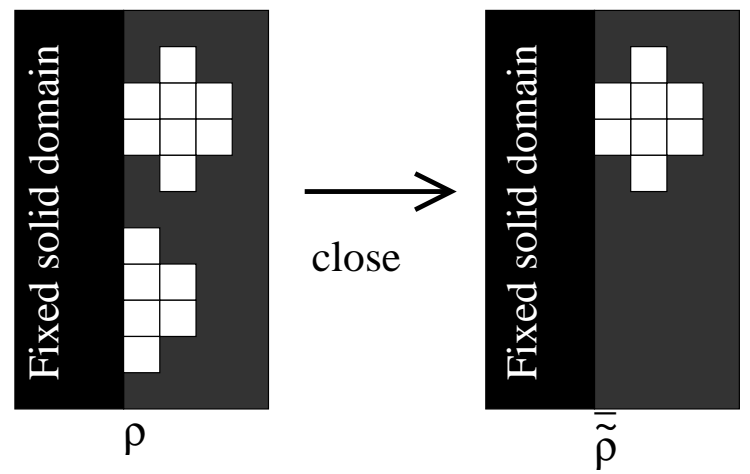

Figure 10: Demonstration of problems with non-volume-preserving filters and fixed solid domains. a) Design domain with fixed solid region and indication of neighborhood size. b) Left: image of design variable field and right: filtered density field after erosion operation. The width of the lower hole is smaller than the neighborhood diameter. c) Left: image of design variable field and right: filtered density field after close operation. The small scale lower hole has been eliminated using the close filter which is volume preserving. 
have been tested on three different test examples and have been compared with methods and modified methods from the literature.

With the check list in section 1 in mind, it must be concluded that the new morphology based filters together with the Heaviside filters provide interesting new methodologies for topology optimization with manufacturing constraints. The only check point that is not fulfilled is point 6: "Stable and fast convergence". Due to the required continuation approach for the $\beta$ parameter, the proposed methods typically use between 500 and 1000 iterations to converge. Whereas this number may be satisfactory from an academic point of view, it will not be accepted for industrial applications, hence the ideal restriction method probably still remains to be invented.

As a suggestion for future work, it is recommended to implement and test the extension of the Heaviside filters (basic version proposed in Guest et al (2004)) to be used as morphology operators, i.e. sequentially applying the Heaviside and the modified Heaviside schemes in order to make open and close operators. It is expected that this will result in a stable convergence to more discrete solutions than in the proposed Kreisselmeier-Steinhauser min-max formulation.

Other possible extensions include anisotropic filters, e.g. by selecting oblong instead of circular structuring elements one may favor certain angles of features in the optimized topologies (for application in architecture). Another interesting possibility is to use the morphology-based operators for perimeter control. The difference between the design variable image $\boldsymbol{\rho}$ and the filtered (eroded or dilated) image $\tilde{\boldsymbol{\rho}}$ correspond to the contour of the topology and hence the number of elements making up the contour is a measure of the perimeter. Finally, there is an option for using different structural elements for the open and close operators which will make it possible to impose different minimum length-scales for holes and structural features. All these extensions are left open for future research.

\section{Acknowledgements}

The author is thankful for valuable discussion on filtering methods with the following individuals: Martin P. Bendsøe, Tyler E. Bruns, James Guest, Jakob S. Jensen, Anders A. Larsen and Pauli Pedersen. This work received support from the Eurohorcs/ESF European Young Investigator Award (EURYI, www.esf.org/euryi) through the grant "Synthesis and topology optimization of optomechanical systems", the New Energy and Industrial Technology Development Organization project (NEDO, Japan), and from the Danish Center for Scientific Computing (DCSC).

\section{References}

Allaire G, Jouve F, Toader AM (2004) Structural optimization using sensitivity analysis and a level-set method. Journal of Computational Physics 194(1):363-393

Ambrosio L, Buttazzo G (1993) An optimal design problem with perimeter penalization. Calculus and Variation 1:55-69

Bendsøe MP (1989) Optimal shape design as a material distribution problem. Structural Optimization $1: 193-202$

Bendsøe MP (1995) Optimization of Structural Topology, Shape and Material. Springer Verlag, Berlin Heidelberg

Bendsøe MP, Sigmund O (2003) Topology Optimization - Theory, Methods and Applications. Springer Verlag, Berlin Heidelberg, XIV+370 pp.

Borel PI, Frandsen LH, Harpøth A, Kristensen M, Jensen JS, Sigmund O (2005) Topology optimised broadband photonic crystal Y-splitter. Electronics Letters 41(2):69-71 
Borrvall T (2001) Topology optimization of elastic continua using restriction. Archives of Computational Methods in Engineering 8(4):351-385

Borrvall T, Petersson J (2001) Topology optimization using regularized intermediate density control. Computer Methods in Applied Mechanics and Engineering 190:4911-4928

Bourdin B (2001) Filters in topology optimization. International Journal for Numerical Methods in Engineering 50(9):2143-2158

Bourdin B, Chambolle A (2003) Design-dependent loads in topology optimization. ESAIM: Control, Optimisation and Calculus of Variations 9:19-48

Bruns TE, Tortorelli DA (2001) Topology optimization of non-linear elastic structures and compliant mechanisms. Computer Methods in Applied Mechanics and Engineering 190(26-27):3443-3459

Bruns TE, Tortorelli DA (2003) An element removal and reintroduction strategy for the topology optimization of structures and compliant mechanisms. International Journal of Numerical Methods in Engineering 57(10):1413-1430

Cardoso EL, Fonseca JSO (2003) Complexity control in the topology optimization of continuum structures. Journal of the Brazilian Society of Mechanical Science and Engineering XXV(3):293-301

Guest J, Prevost J, Belytschko T (2004) Achieving minimum length scale in topology optimization using nodal design variables and projection functions. International Journal for Numerical Methods in Engineering 61(2):238-254

Guo X, Gu YX (2004) A new density-stiffness interpolation scheme for topology optimization of continuum structures. Engineering Computations: Int J for Computer-Aided Engineering 21(1):922

Haber RB, Jog CS, Bendsøe MP (1994) Variable-topology shape optimization with a constraint on perimeter. In: Gilmore B, et al (eds) Advances in Design Automation, ASME, New York, vol DE 69-2, pp 261-272

Jensen JS, Sigmund O (2004) Systematic design of photonic crystal structures using topology optimization: Low-loss waveguide bends. Applied Physics Letters 84(12):2022-2024

Jensen JS, Sigmund O (2005) Topology optimization of photonic crystal structures: A high-bandwidth low-loss T-junction waveguide. Journal of the Optical Society of America B: Optical Physics 22(6):1191-1198

Kim YY, Yoon GH (2000) Multi-resolution multi-scale topology optimization - a new paradigm. International Journal of Solids and Structures 37:5529-5559

Kreisselmeier G, Steinhauser R (1983) Application of vector performance optimization to a robust control loop design for a fighter aircraft. International Journal of Control 37(2):251-84

Niordson FI (1983) Optimal design of plates with a constraint on the slope of the thickness function. International Journal of Solids and Structures 19:141-151

Petersson J, Sigmund O (1998) Slope constrained topology optimization. International Journal for Numerical Methods in Engineering 41(8):1417-1434

Poulsen TA (2002) Topology optimization in wavelet space. Int Journal Numerical Methods in Engineering 53:567-582

Poulsen TA (2003) A new scheme for imposing a minimum length scale in topology optimization. Int Journal Numerical Methods in Engineering 57(6):741-760 
Pratt WK (1991) Digital Image Processing. John Wiley and Sons, New York

Sethian JA, Wiegmann A (2000) Structural boundary design via level set and immersed interface methods. Journal of Computational Physics 163(2):489-528

Sigmund O (1994) Design of material structures using topology optimization. PhD thesis, Department of Solid Mechanics, Technical University of Denmark, DK-2800 Lyngby, the Danish Center for Applied Mathematics and Mechanics, DCAMM Special Report No. S69

Sigmund O (1997) On the design of compliant mechanisms using topology optimization. Mechanics of Structures and Machines 25(4):493-524

Sigmund O (2000) Topology optimization: A tool for the tailoring of structures and materials. Philosophical Transactions - Mathematical Physical and Engineering Sciences 358(1765):211-228

Sigmund O (2001a) A 99 line topology optimization code written in MATLAB. Structural and Multidisciplinary Optimization 21:120-127, DOI 10.1007/s001580050176, mATLAB code available online at: www.topopt.dtu.dk

Sigmund O (2001b) Design of multiphysics actuators using topology optimization - Part II: Twomaterial structures. Computer Methods in Applied Mechanics and Engineering 190(49-50):66056627, DOI 10.1016/S0045-7825(01)00252-3

Sigmund O, Jensen JS (2003) Systematic design of phononic band gap materials and structures by topology optimization. Philosophical Transactions of the Royal Society A: Mathematical, Physical and Engineering Sciences 361:1001-1019

Sigmund O, Petersson J (1998) Numerical instabilities in topology optimization: A survey on procedures dealing with checkerboards, mesh-dependencies and local minima. Structural Optimization 16(1):68-75

Svanberg K (1987) The Method of Moving Asymptotes - A new method for structural optimization. International Journal for Numerical Methods in Engineering 24:359-373

Wang M, Zhou S (2004) Phase field: A variational method for structural topology optimization. CMES - Computer Modeling in Engineering and Sciences 6(6):547-566

Wang M, Wang X, Guo D (2003) A level set method for structural topology optimization. Computer Methods in Applied Mechanics and Engineering 192(1-2):227-246

Wang MY, Wang S (2005) Bilateral filtering for structural topology optimization. International Journal for Numerical Methods in Engineering 63(13):1911-1938

Zhou M, Shyy YK, Thomas HL (2001) Checkerboard and minimum member size control in topology optimization. Structural and Multidisciplinary Optimization 21:152-158 